\title{
Determinación de orientaciones de ciudades romanas por medio de la varatio* $^{*}$
}

\section{Orientation layout of Roman towns by using the varatio}

\author{
Margarita Orfila Pons ${ }^{1}$ \\ Universidad de Granada \\ Andrea Rodríguez-Antón ${ }^{2}$ \\ Instituto de Ciencias del Patrimonio, Incipit-CSIC \\ Esther Chávez-Álvarez ${ }^{3}$ \\ Universidad de La Laguna \\ Antonio César González-García ${ }^{4}$ \\ Instituto de Ciencias del Patrimonio, Incipit-CSIC \\ Elena H. Sánchez López ${ }^{5}$ \\ Universidad de Granada \\ Juan Antonio Belmonte ${ }^{6}$ \\ Instituto de Astrofísica de Canarias y Universidad de La Laguna
}

\section{RESUMEN}

En el presente artículo se aborda la tan debatida cuestión de las orientaciones de ciudades romanas combinando dos procedimientos: un análisis estadístico de las orientaciones de una muestra significativa de ciudades romanas en la península

\footnotetext{
* Este trabajo se enmarca dentro de los proyectos de investigación del Plan Nacional de I+D+i "Un sistema para orientar y trazar las estructuras ortogonales de época romana. Su identificación en Pollentia, su aplicación en otros yacimientos (Sotoer)" (HAR2009-11824, subprograma HIST) y "La Varatio y sus variaciones. La homologación en el proceso del trazado y orientación de estructuras ortogonales en época romana y sus precedentes (Sotoer 2)" (HAR2013-41635-P), los proyectos P/310793 "Arqueoastronomía” del IAC, (AYA2011-26759) "Orientatio ad Sidera III" y (AYA2011-66787-P) "Orientatio ad Sidera IV" del MICIN. Además, queremos expresar nuestro agradecimiento por la contribución del proyecto: Desarrollo de instrumental topográfico de época romana y su aplicación en la actualidad (MP-CP-5-2014, CEI-Biotic, UGR), y a todo el equipo del Grupo de Investigación GAEGATAO HUM-296.

orfila@ugr.es / ORCID iD: http://orcid.org/0000-00031773-998X

2 rodriguezantonandrea@gmail.com / ORCID iD: http:// orcid.org/0000-0002-0786-3963

3 echavez@ull.edu.es / ORCID iD: http://orcid.org/00000002-0687-0360

${ }^{4}$ a.cesar.gonzalez-garcia@incipit.csic.es / ORCID iD: http:// orcid.org/0000-0003-1831-8562

5 elenasanchez@ugr.es / ORCID iD: http://orcid.org/00000002-7807-9770

${ }^{6}$ jba@iac.es / ORCID iD: http://orcid.org/0000-0002-8357-
}

ibérica, y los resultados de una investigación basada en la experimentación y reconstrucción de instrumental de agrimensura romana. En concreto, se busca reforzar, o rechazar, la hipótesis del empleo de la varatio para la orientación en el terreno, y su posible modo de ejecución: método basado en la aplicación de triángulos rectángulos con catetos en proporción de números naturales. Se postula además la línea meridiana como la generadora de cada nuevo proyecto, incluyéndose un posible procedimiento para su obtención a partir de los preceptos de Vitruvio (De Architectura VI, I). Los resultados del análisis estadístico devuelven una distribución de las orientaciones no aleatoria y que, en muchos casos, está en buen acuerdo con los valores predichos de la aplicación de la técnica de la varatio en sus diferentes variantes. Con esto se pretende determinar si sería factible el empleo de una técnica estandarizada para trazar los patrones de orientación obtenidos.

\section{SUMMARY}

This paper deals with the long-lasting debate about the orientation of Roman cities by combining two procedures: an analysis of a statistically significant sample of orientations of Roman settlements in the Iberian Peninsula and the results of the experimentation and re-construction of Roman surveying instruments. Specifically, the study seeks to reinforce, or reject, the hypothesis of the use of the varatio, and its possible mode of execution: a method based on the application of right-angled triangles with legs in proportion of integer numbers. The meridian line is also postulated as the generating line for each new project, including a possible procedure for obtaining it

Copyright: ( 2020 CSIC. Este es un artículo de acceso abierto distribuido bajo los términos de una licencia de uso y distribución Creative Commons Reconocimiento 4.0 Internacional (CC-by 4.0). 
from the indications given by Vitruvius (De Architectura VI, I). The results derived from the statistical analysis show that orientations seem not to be randomly distributed but they tend to cluster around particular directions that agree with the expected values in case this geometrical technique was commonly applied.

PALABRAS CLAVE: Hispania; urbanismo romano; agrimensura; geometría; análisis estadístico.

KEY WORDS: Hispania; Roman urbanism; land surveying; geometry; statistical analysis.

CÓMO CITAR ESTE ARTÍCULO / CITATION: Orfila Pons, M., Rodríguez-Antón, A., Chávez-Álvarez, E., GonzálezGarcía, A. C., Sánchez López, E. H. y Belmonte, J. A. 2020: "Determinación de orientaciones de ciudades romanas por medio de la varatio", Archivo Español de Arqueología 93, 127-146. https://doi.org/10.3989/aespa.093.020.006

\section{INTRODUCCIÓN}

La división del terreno era una parte esencial del proceso de expansión y ordenación territorial romano tras una nueva conquista, en la que se incluía la construcción de nuevas parcelaciones, o entes urbanos, especialmente si se trataba de la implantación de una colonia. Para ese menester se requería de responsables oficiales de la obra, como el curator operis que lo coordinaba todo, el architectus, mensor o librator que definía la planta, siendo los gromatici o agrimensores los que marcaban in situ los límites, la medida de las parcelas a crear, y plasmaban su orientación (Lewis 2001). Los tratados de estos expertos están recopilados en el Corpus Agrimensorum Romanorum. Una de las formas más comunes de delimitar el terreno era mediante centuriaciones, que consistían en divisiones cuadradas o rectangulares desarrolladas a partir de un sistema de ejes ortogonales. En la creación de estas parcelaciones entraría la planificación y creación del espacio urbano -normalmente basado en un diseño ortogonal-, precedido de un ritual de inauguración, liderado por un augur, papel que solía asumir el conditor, el magistrado responsable de la fundación, praefectus. Por otra parte, si se concibe el territorio, o territorium, como un espacio dotado de significado, el conocimiento de los modelos de ordenación de esos espacios debería favorecer la comprensión de los procesos históricos de los que resultaron estos. La combinación de principios de practicidad con una voluntad ritual en la ejecución de las obras (Orfila et alii 2017a), conduce a un inevitable planteamiento acerca de las motivaciones que llevaron a elegir las orientaciones de los parcelarios, así como de las técnicas empleadas para su desarrollo. Sobre esta última cuestión trata el presente artículo. Puesto que existen varias posibili- dades e hipótesis sobre cómo los romanos conseguían orientar una ciudad, la cuestión que se nos plantea es si las medidas obtenidas de la orientación de un amplio número de ciudades romanas en la península ibérica se pueden explicar por medio de un método estandarizado ya explorado en trabajos previos: la varatio y su variante con ternas pitagóricas (Orfila y Moranta 2001; Orfila 2012; Equipo Sotoer 2014, Orfila et alii 2017b). Por este motivo, para establecer si este procedimiento se empleaba a la hora de orientar un nuevo asentamiento, se impone realizar una comparación empírica de las orientaciones de un número significativo de ciudades romanas en la península ibérica con los ángulos teóricos que se pueden obtener al aplicar estas técnicas geométricas.

\section{DETERMINACIÓN DE ORIENTACIONES SOBRE URBANIZACIONES Y EDIFICIOS EN ÉPOCA ROMANA: CAUSAS}

En época romana el ideal de la orientación de los viales y edificios era que coincidieran con los puntos cardinales. Recordemos la importancia manifiesta de alinear los ejes principales en las urbanizaciones, el decumano máximo y el cardo máximo, superpuestos, respectivamente, a la línea equinoccial y la meridiana. Una orientación que suponía, además, un símbolo de respeto hacia los dioses, dado que de esta manera se imitaba en tierra la estructura de su morada, el templum caelestis (Chevallier 1967: 6; Castillo Pascual 1993: 144). El cruce de estas líneas asumía el componente ritual y simbólico de la ortogonalidad (Ventura 2008). Un simple repaso a las viñetas que acompañan los escritos de los Gromatici Veteres permite identificar esa figura reiteradamente. Buen ejemplo es el de la Colonia Iulia mencionada por Higinio Gromático y plasmada en el manuscrito Arcerianus A -Wolfenbüttel, Herzog August Bibliothek- (Chouquer y Favory 2001: 282, fig. 17), en donde las vías urbanas y rurales coinciden con esas alineaciones cardinales. Era el paso de un espacio natural a otro antropizado (Rosada 1991), planificado según el diseño de la mente humana, y sometido, siempre prudentemente, a los designios divinos, tal como Higinio Gromático señaló (Hyg. Grom. De lim. Cons. 170.5).

Sin embargo, la realidad empírica parecería indicar que la orientación era determinada por causas relacionadas con el uso y funcionalidad de las infraestructuras a crear, y estaba condicionada por la orografía del terreno (Orfila y Chávez-Álvarez 2014; Orfila et alii 2017b). En este sentido, es significativo el capítulo primero del libro sexto de Vitruvio, dedicado íntegramente a la orientación, en donde señala 
que un criterio importante a tener presente al iniciar las obras está estrechamente vinculado con el clima, la salubridad y el propio lugar. Vitruvio indicó que las alineaciones elegidas para los viales de la ciudad dependían de los vientos reinantes, puesto que consideraba insano que las calles canalizaran las corrientes que estos producían (Vitruvio VI, I). También aconsejaba sobre la importancia del grado de insolación necesario en los locales del mercado de los foros, para no sufrir las inclemencias del tiempo (Vitruvio VI, I). Por eso los comedores de invierno de las casas y sus salas de baños debían mirar al poniente invernal, pues así tendrían claridad vespertina, y los dormitorios y bibliotecas hacia levante (Vitruvio VI, VII). Varrón también escribió en ese sentido: "La más ventajosa es la orientación hacia levante, que es muy propia para tener sombra en el verano y sol en el invierno" (Varrón I, 12, 1). Estas recomendaciones también recaen en la necesidad de calidez en la zona de almacenamiento de aceite en una villa, o evitarla en el caso del vino (Vitruvio VI, IX). Consejos de orientación que alcanzan a otras construcciones, como los templos (Vitruvio IV, v), en ese caso con una clara intencionalidad cultual, o la necesidad de sol incidiendo en las termas (Vitruvio $\mathrm{V}, \mathrm{XI})$, por poner algún otro ejemplo.

La figura inicial en forma de cruz también tiene otro cariz pues uno de sus ejes se relaciona con el recorrido del sol en los equinoccios, señalando su orto y ocaso, respectivamente, el este y oeste en el momento del cambio de estación, ya sea en el paso de invierno a primavera ( 20 o 21 de marzo) o con la llegada del otoño ( 22 o 23 de septiembre). Ambos posicionamientos son de carácter universal puesto que, en esos días, el sol sale y se pone a $90^{\circ}$ en relación con la línea meridiana en cualquier punto del globo terráqueo siempre que el horizonte está despejado y su altura angular sea de $0^{\circ}$. Por otro lado, debe tenerse también en cuenta que, al contrario que en los equinoccios, el acimut de los ortos y ocasos en los solsticios varía en función de la localización geográfica y de la altura del horizonte. Esta variabilidad queda evidenciada en la amplitud del rango de acimut entre ambos ortos solsticiales -el arco comprendido entre las salidas (o puestas) en los solsticios de verano e invierno- en las latitudes a las que se encuentra la franja abarcada por el Imperio Romano, que oscila entre latitud $23^{\circ}$ en la frontera egipcia del Imperio y latitud $54^{\circ}$ aproximadamente en el muro de Adriano (Costa y Orfila 2014: 152-155, fig. 76; Belmonte y González-García 2013) (Fig. 1). A esto habría que sumar el efecto que produce la altura del horizonte local en la visibilidad de los astros. Además, el valor del ángulo de inclinación del eje de rotación de la Tierra respecto al plano de su órbita alrededor al sol varía a largo plazo debido a efectos gravitacionales, lo que influye en la amplitud de dicho rango de acimut entre los ortos solares de ambos solsticios. Desde época romana hasta hoy en día la amplitud de este rango ha disminuido alrededor de $14^{\circ}$ de acimut en cada extremo solsticial en latitudes medias de la península ibérica; y entran, por tanto, dentro del margen de error estimado para nuestros datos (Ruggles 2015a: 479-481).

Los ortos y ocasos del sol, los equinoccios y solsticios, fueron importantes como puntos de referencia hacia donde orientar alineaciones urbanas (Gottarelli 2003: 156, fig. 3; Gottarelli 2004: 142-143; GonzálezGarcía y Magli 2015: 65-66; González-García et alii 2014: 107-119; Rodríguez-Antón et alii 2018b). Estos posicionamientos funcionaron como marcadores temporales, convirtiéndose esas construcciones en calendarios vitales para el reconocimiento de los cambios estacionales u otras fechas relevantes. Un ejemplo significativo de la importancia de los solsticios puede observarse en el trazado urbano de Carthago Nova (González-García et alii 2015) o en el propio Panteón de Roma. En este último, arquitectura y astronomía se combinan, no tanto con la finalidad de realizar mediciones de tiempo precisas, sino para indicar la conexión simbólica entre el edificio, el tiempo y la trayectoria del sol en el curso del año (Hannah y Magli 2011; Lanciano y Virgili 2016).

En otras ocasiones se ha interpretado que los trazados estuvieran orientados a posiciones del sol en otros días especiales. Entre ellos, la conmemoración del dies natalicius urbis (Ekcstein 1979), el del nacimiento de la propia ciudad. Así, en Roma, las orientaciones de los edificios del Area Sacra de Largo Argentina y el teatro de Pompeyo, están alineados según la puesta del sol del 21 de abril, mientras que la Porticus Liviae lo está al alba del mismo día (Capone 1991: 60), fecha mítica de la fundación de la Urbs por Rómulo. En esta misma línea pudieron ser utilizados el día del nacimiento de Augusto, como se propone para la orientación del Ara Ubiorum (Colonia) (Espinosa Espinosa y González-García 2017), o del fundador de una ciudad determinada. Se ha propuesto que en la ciudad helenística de Alejandría se alineó el eje principal de la ciudad, la vía Canópica, con el orto del sol en el probable día del nacimiento de Alejandro Magno, el 20 de julio (Ferro y Magli 2012: 387).

Los posicionamientos lunares tampoco se descartan como origen de algunos alineamientos, y de hecho, Vitruvio también recurrió a ellos como modo de contabilización del tiempo (Vitruvio IX, II). En otros casos, las interpretaciones astronómicas asocian las

${ }^{7}$ Para un breve repaso sobre astronomía de posición consultar el trabajo de Ruggles (2015b: 459-471). 

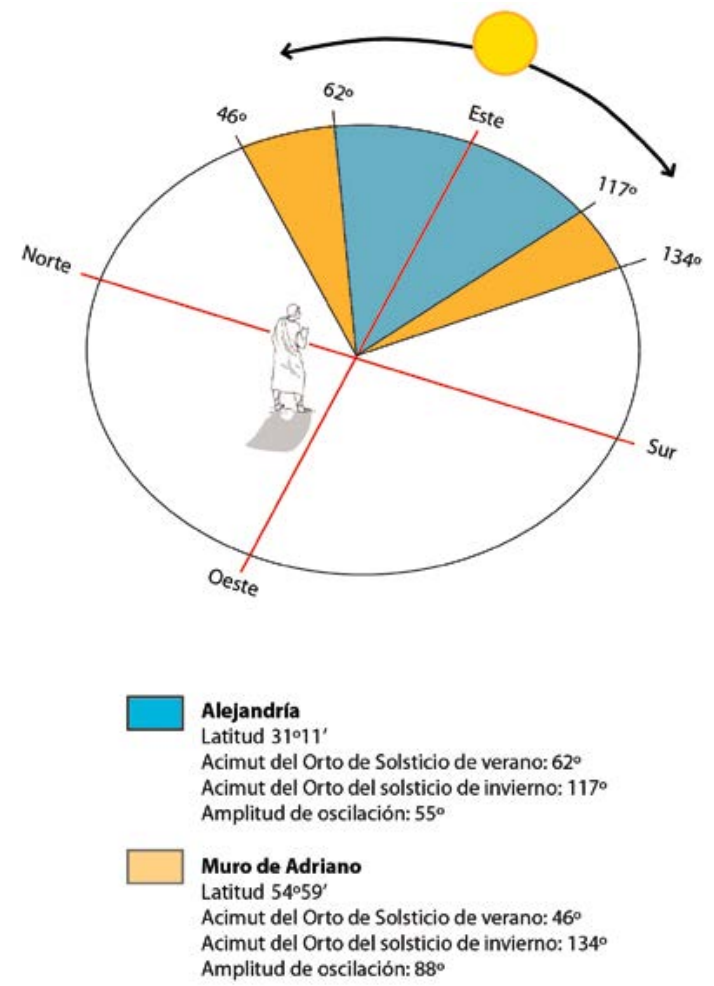

Figura 1. Representación de las diferencias en ortos del sol entre la zona de Alejandría y la del Muro de Adriano para un horizonte plano (Costa y Orfila 2014: 154).

orientaciones a puntos en donde aparecen determinadas estrellas en el firmamento. Una de ellas sería Sirio, con la que se relaciona la orientación de la ciudad de Sabratha, en Tripolitania (Esteban et alii 2001; Belmonte y Hoskin 2002), y probablemente también la de Sufetula en el Africa Proconsularis (Belmonte et alii 2006: 77-79).

Pero estas no fueron las únicas causas que determinaron las orientaciones. Tal como ya se ha insinuado más arriba, cuestiones de tipo práctico, como las pendientes, adaptarse a las curvas de nivel, vías de comunicación preexistentes, ríos o líneas de costa y montaña, facilitar los drenajes y que el agua corra gracias a la acción de la gravedad, entre otras cuestiones, también se tuvieron en cuenta para elegir la orientación adecuada de muchas obras (Le Gall 1975: 309). Autores como Frontino, Higinio Gromático o Pseudo Higinio, relacionaron criterios como estos a la hora de decidir sobre la orientación a conferir a las divisiones rurales (Rossella Filippi 1983: 125-126; Orfila y Chávez-Álvarez 2014a: 64; Orfila et alii 2017b).

Influyeron, por ejemplo, razones de estrategia militar a la hora de implantar un campamento para poder desarrollar tácticas militares en contra del enemigo, o de tipo nodal si era una ciudad de carácter comercial, al conferir, por su orientación, la visualización de las vías de comunicación por donde se circularía. Por otra parte, la vecindad entre ager diuisus et adsignatus de dos demarcaciones administrativas diferentes, como indicó Higinio Gromático, obligaba a que cada centuriatio determinara orientaciones distintas para distinguirlas claramente entre sí (Hyg. Grom. De lim. Cons. 170) (Rossella Filippi 1983: 126).

Frente a estos planteamientos, existen también voces que indican que esas orientaciones dispares fueron producto del azar. Es el caso de Peterson (2007), quien señala, en la publicación titulada muy explícitamente Random Orientation of Roman Camps, que la coincidencia de orientaciones no fue debida a la aplicación de una misma técnica y con unos mismos criterios, sino que fueron producto del azar (Peterson 2007: 106).

\section{LA DETERMINACIÓN DE LAS ORIENTACIONES: CONDICIONANTES Y RESTRICCIONES}

No parece que la orientación de las estructuras se dejara al azar, como tampoco parece muy práctico, salvo casos excepcionales, que para calibrar y revisar las alineaciones de, por ejemplo, una centuriación con una groma, se debiera esperar un año en el caso de haberse tomado como referencia el orto del sol en un día en concreto. Y eso si ese día no amanecía nublado (Orfila y Chávez-Álvarez 2014: 118) ${ }^{9}$. Ya indicó Higinio Gromatico: "Multi ignorantes mundi rationem solem sunt secuti, hoc est ortum et occasum, quod is semel ferramento comprehendi non potest. Quid ergo?", cuando señalaba que se equivocan los que se dejan guiar por el movimiento del sol, es decir, tomando como referencias su salida y/o puesta y colocando la groma de acuerdo a esa premisa (La. 170, 3-8, fig. 134 = Th.135, 1-6) (Dilke 1971: 57; Le Gall 1975: 304; Chouquer y Favory 1992: 68), dado que solo los equinoccios son los que marcan el este y el oeste con un horizonte plano.

Los agrimensores, identificados como mensores agrorum o agrarii (Baratta 2006: 236-244), también lo fueron bajo el apelativo de gromáticos, asumido

\footnotetext{
8 Trabajos muy discutidos por Richardson (2005) y posteriormente por Rodríguez-Antón (Rodríguez-Antón et alii 2016a).

9 Si bien no sería algo difícil de calcular en época romana a partir de los conocimientos astronómicos que se poseían. Ejemplo de ello son las referencias que hace Vitruvio (De Architectura $\mathrm{I}, \mathrm{x})$ acerca de la importancia de la astronomía para la confección de relojes solares adecuados para cada lugar.
} 
historiográficamente debido al uso de la groma, el instrumento empleado para llevar a cabo sus operaciones (Adam 1982; Chouquer y Favory 2001: 66-67; Orfila et alii 2014b: 126-129). Las figuras obtenidas con ella, de componente axial, han marcado, de una manera u otra, la estructuración de la mayoría de las obras del mundo romano (Behrends 1992), cuyo resultado son estructuras en cuadrículas. Frontino lo dejó por escrito "...debet enim minima quaeque pars agri in potestate esse mensoris et habitura rectorum angulorum ratione sua postulatione constrigi" (Thulin 1913, Th. 16, 80), es decir, que cada mínima parte del campo debía estar a disposición del mensor y sometido a lo que exigía el método de los ángulos rectos, única forma para conseguir superficies ortogonales, en retícula.

Pero la groma tenía sus limitaciones. Son varios los autores que han tratado este tema: como Adam (1982), Toro (1983 y 1985), Chouquer y Favory (1992: 65-76) o Campbell (1996: 84). Las recreaciones que se han hecho del instrumento desde el Equipo Sotoer, enmarcadas en el desarrollo de proyectos de investigación subvencionados por el Ministerio de Economía y Competitividad, la Universidad de Granada y el Parque de las Ciencias de esta ciudad, así como la experimentación con apoyo de réplicas de otras piezas que acompañaban a la misma, como metae, perticae, etc. (Chouquer y Favory 2001: 65-71), nos han permitido estimar el alcance de la groma, que no sobrepasa los cuarenta metros si se quiere señalar con precisión un punto perfectamente alineado. Eso es consecuencia del llamado efecto de viñeteo, que dificulta la visualización de las metae de manera nítida a partir de esa distancia (Orfila et alii 2014b: 126-129). En este sentido, es importante recordar que en época romana no se tenía la ayuda de la óptica, como sí se tiene desde hace unos siglos. En consecuencia, lo máximo que se podría señalar son superficies que no sobrepasaran los 80 metros de lado (Orfila et alii 2017b) ${ }^{10}$. No debe extrañar, por tanto, que las medidas básicas de extensión de superficie sean múltiplos de un actus cuadrado, área cuyo lado mide 120 pies, correspondientes a unos 35,5 metros. El múltiplo, un iuguerum, son dos actus, mientras que cuatro es un heredium (Chouquer y Favory 2001: 75-78; Salvatori 2006). Dado que la groma funcionaba ubicándola en el centro de un cruce perpendicular de alineaciones, quiere decir que el alcance total se multiplica por dos. En ese caso, al ubicarla en el centro de un heredium, desde cada brazo

${ }^{10}$ En las pruebas que hemos realizado se pudieron señalar puntos cercanos a los 90 metros, acumulando errores, pero con cierta precisión, pudiéndose visualizar las metae a unos 140, ya sin exactitud (Orfila et alii 2017b). se podía señalar con precisión un actus, alcanzando de punta a punta 240 pies, (cuatro actus quadratus), equivalentes a $71 \mathrm{~m}$ de lado.

Al trabajar en tramos cortos, como los señalados en el párrafo anterior, no extraña que se perciban hoy variaciones mínimas de sus orientaciones entre cada uno de ellos. A ese efecto debe añadirse el paso del tiempo, que siempre va deformando las alineaciones. Es por ello que, en una construcción reflejada sobre planimetría a escala 1:20, puede dar la sensación de que cada uno de esos tramos tiene una alineación diferente entre sí, pero, el resultado final de toda la obra realmente sí sigue una misma alineación a larga escala. Ese hecho nos acerca a la realidad del acabado de las obras, no siempre perfecto (Dall'Aglio 2004).

A las limitaciones del instrumental, debe sumarse otra cuestión: el modo de cálculo utilizado en época antigua. Al basarse en una contabilización a partir de unidades, es evidente que determinados ejercicios no se podían realizar con precisión. De ahí que, tal como indicó Gros (1976), los números irracionales se representaran con valores de aproximación a través de fracciones (Neugebauer 1969); de hecho, los romanos utilizaban fracciones en todo tipo de cuentas (Peterson 1992). Si de nuevo recurrimos a Vitruvio, se tienen muy buenos ejemplos de ese uso de fracciones en las relaciones que él mismo propone para conseguir superficies armónicas. El templo toscano debe ser equivalente en sus lados a la relación 6:5 (IV, VIII), 2:3 para los foros (V, I), 1:2 o 1:3 para las basílicas (V, I), y las opciones 3:5 y 3:2 para las medidas de los atrios (VI).

En el caso de calibrar el desvío de alineaciones desde una línea de referencia, el modo de cálculo no pudo ser otro que el de fracciones, que es lo que aplica Marcus Iunius Nypsus en el siglo II d. C. -si bien es posible que se aplicase con anterioridad-al describir la técnica de la varatio $^{11}$, utilizando la figura geométrica de los triángulos rectángulos. Esta explicación aparece dentro del conjunto de obras que conforman el Corpus de los Gromatici Veteres, del que Chouquer (2004) realizó una nueva interpretación al asociar su redacción al catastro que hubo de realizarse tras el incendio del archivum de Roma. Así, Nypsius explica cómo identificar los limites de unas parcelaciones de un ager diuisus et adsignatus con otras, a través de la identificación de la ratio a la que se orientaron cada una de ellas, es decir, a través de la varatio.

11 Técnica descrita por Nypsius en Fluminis varatio y Limitis repositio (La. 285.1-295.15; Bouma 1993). Un estudio exhaustivo de la misma en Roth-Congès (1996 y 1998). 


\section{PROPUESTA DE PROCEDIMIENTO DE PLASMACIÓN SOBRE EL TERRENO DE UNA ORIENTACIÓN DETERMINADA}

Según nuestra propuesta, evidentemente no dogmática, pero sí resolutiva en muchos casos, el primer paso que debía darse para determinar una orientación era precisamente localizar esa línea generadora gracias a la ayuda de un gnomon. Este era el instrumento que autores clásicos como Vitruvio (IX, I y VI) e Higinio Gromático (La. 188.17 - 189.15; Th. 152.7-22), recomendaban utilizar para ese menester (Castillo Pascual 1996: 90). Su modo de uso ha sido reproducido en diversas publicaciones (Le Gall 1975: 304, fig. 304; Rossella Filippi 1983: 126, fig. 97; Haselberger 1999, fig. 3; Chouquer y Favory 2001: 90, fig. 85-86; Gottarelli 2003: 157, fig. 4; Orfila 2011, figs. 1-3; Orfila et alii 2014a: 125, fig. 60).

Si no era conveniente alinear la obra a los puntos cardinales, se determinaba la orientación elegida utilizando la técnica de la varatio (Roth Congés 1996; Orfila 2012; Equipo Sotoer 2014; Orfila et alii 2017b). En este caso, se tomaría un triángulo rectángulo con los catetos en proporción de números enteros y se colocarían estos a lo largo de los ejes cardinales. Según la ratio elegida entre las unidades dadas a cada uno de los catetos, varía la orientación respecto al norte, dependiendo de la hipotenusa resultante (Fig. 2A: 3a, 3b), sobre la que se podría trazar la línea perpendicular mediante su bisectriz. Otra manera de trazar la perpendicular sería alineando uno de los ejes de la groma con la hipotenusa resultante de modo que el otro eje daría la orientación ortogonal. De este modo se establecería una nueva cruceta cuyo punto central se convertía en el locus gromae del trazado (Fig. 2A: 3 a y $3 b$ ). Estas correlaciones de fracciones alcanzan un total de 47 posibilidades por cuadrante de circunferencia si se consideran fracciones desde 1:1 hasta 12:12 (Equipo Sotoer 2014: 82, fig. 30) (Fig. 2B). Pero, debido a la simetría intrínseca del cálculo de ángulos de la varatio, al recrearse solares de forma cuadrangular o rectangular, del total de los $360^{\circ}$ de una circunferencia, debe restringirse a un octavo, que son $45^{\circ}$, reduciéndose ese número de posibilidades al fusionar orientaciones opuestas, como por ejemplo 1:3 y 3:1. Al mismo tiempo, cada fracción tiene su equivalente en grados (Equipo Sotoer 2014: 85) ${ }^{12}$, luego se ha de evitar repetir ángulos iguales, como serían los resultantes

12 Tablas semejantes, pero más sencillas, están presentes en las obras de Guy (1993: 63, fig. 6) y Peterson (1992: fig. 4). De hecho, Clavel-Levêque propuso que los agrimensores debían utilizar unas tablas con las fórmulas y sus equivalencias (ClavelLevêque 1992: 175). de relaciones equivalentes como 1:3 y 3:6 o el ángulo de $45^{\circ}$, resultante de la fracción 1:1, y sus múltiplos (Roth Congès en Chouquer y Favory 2001: 91). El número de opciones podría aumentar si superamos la fracción 12:12, consiguiendo, un número más elevado de opciones, y, al mismo tiempo, una mayor complejidad en cuanto a su aplicación, además de, en casos de valores muy elevados, una pérdida de exactitud en los cálculos, lo que no parece operativo si se tiene en cuenta el alcance del instrumental utilizado por los gromáticos.

El modo de comprobación de la exactitud del ángulo en $90^{\circ}$ en la conjunción de los catetos se llevaba a cabo aplicando al valor de los catetos e hipotenusa coincidente con una terna pitagórica, una práctica que se denominaba pro hipotenusa (Guy 1993: 59 y 62; Pérez 1995: 51-52). En esos casos se podría conseguir plasmar trazados con la simple ayuda de un cordel, tal como Guy muestra en su publicación (Guy 1993). Esta práctica fue representada por Higinio Gromático en la explicación del uso de la groma-ad perpendiculumy de las metae (La. 192, 7-193) (Chouquer y Favory

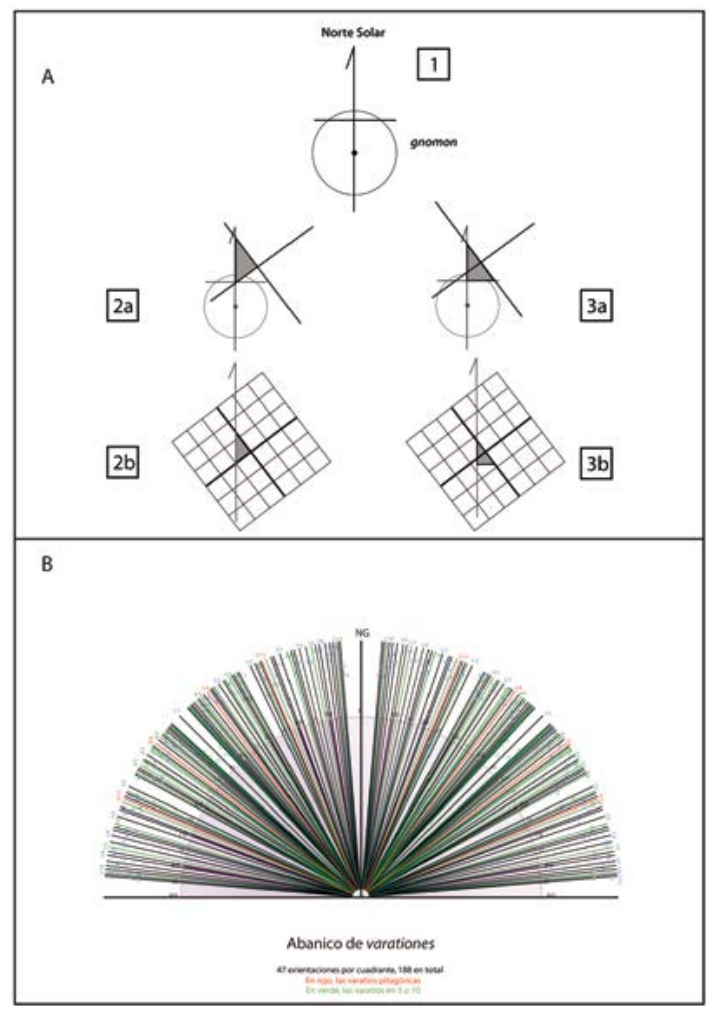

Figura 2. A Representación del procedimiento a través de las ternas pitagóricas $(2 \mathrm{a}, 2 \mathrm{~b})$ y de la varatio $(3 \mathrm{a}, 3 \mathrm{~b})$. B Abanico con las diversas opciones plausibles de ser proyectadas a través de la varatio (elaboración propia). 
1992: 82). De hecho, en la obra Podismus, atribuida por K. Lachmann a Nypsus, y anónima para otros (Chouquer y Favory 2001: 14), aparecen referencias específicas de utilización de ternas pitagóricas, ya sea la 3:4:5 (La. 300, 1-6, viñeta 217) y su múltiplo 6:8:10 (viñetas 218 y 219), la 15:20:25 (La. 297,16 - 298.11, viñeta 214), o 8:15:17 (La. 298,12 - 299,3, viñeta 215) (Roth-Congès 1996: 340).

Por otra parte, si los triángulos rectángulos empleados eran coincidentes con ternas pitagóricas, se podía producir un nuevo modo de ejecución en el proceso de determinación de una orientación. En este caso ubicando la hipotenusa de uno de estos triángulos sobre uno de los ejes cardinales, en principio el nortesur estacionando la groma en la confluencia de los dos catetos-locus gromae-(Fig. 2A: 2a, 2b), reflejo de la orientación que se quería conferir a ese nuevo trazado. Este procedimiento, al que denominamos "por ternas pitagóricas" (Orfila y Chávez-Álvarez 2014a), ya fue propuesto en 2001 (Orfila y Moranta 2001: 232), basándonos principalmente en el uso de las dos ternas más bajas, las 3:4:5 y la 5:12:13, como modo de obtención de alineaciones (Orfila 2009; 2012; Orfila et alii 2014a). En este caso, las posibilidades que ofrece este modo de proceder son menores que las de la propia varatio (Orfila et alii 2014d: 96), y, a la par, coincidentes en el resultado final; la única diferencia es que es factible reproducir la figura de la planta que se quiere desarrollar utilizando un mínimo de instrumentos. De nuevo, las dimensiones del trazado a generar no pueden superar una superficie muy amplia, dado que los errores de cálculo se irían acumulando progresivamente, y no sería operativo.

La distancia a la que se ubicaba cada uno de los gnómones se calibraría mediante la fluminis varatio, descrita por Nypsius (Dilke 1971: 60; Rossella Filipi 1983: 133-134). Es un procedimiento semejante al aplicado en la cultellatio que Frontino explicó en De limitibus (26-27; 33-34) (Rossella Filippi 1983: 134).

\section{COMPROBACIONES SOBRE PLANIMETRÍAS Y RESTOS ARQUEOLÓGICOS}

Tradicionalmente, las líneas que marcan el norte sobre las planimetrías de restos arqueológicos se han obtenido mediante la ayuda de brújulas. Su consecuencia ha sido que lo plasmado en ellas habitualmente haya sido el norte magnético (Le Gall 1975; Romano 1991: 24-26), sin calibrar, en muchas ocasiones, su desviación respecto al norte real, ni indicar la naturaleza de medida magnética de tal coordenada consignada en los mapas. Un error, dado que lo que se tuvo en cuenta en época romana fue el norte geográfico
(Orfila 2009: 295; Orfila 2012: 131-2; Orfila y ChávezÁlvarez 2014: 113) que, a diferencia de lo que sucede con el magnético, básicamente no ha variado.

Al igual que en trabajos previos de arqueoastronomía (Magli 2008; González-García et alii 2014) en el presente artículo también hemos incidido en este tema, que de hecho ha dificultado los trabajos de comprobación del uso de la varatio para la orientación de infraestructuras, dado que en ocasiones no ha sido posible usar como referencia el norte plasmado en las publicaciones por resultar poco fiable (Orfila y Chávez-Álvarez 2014: 112-114). De ahí el planteamiento de los proyectos Sotoer 1 y 2, de reproducir in situ los ejes cardinales con un gnomon en los propios yacimientos (Costa y Orfila 2014). Los resultados de estas comprobaciones, calibrando las orientaciones respecto al norte geográfico (Orfila 2012; Orfila et alii 2014; Sánchez y Marín 2014), permiten afirmar que en todas ellas se ha podido identificar bien el uso de la varatio en la determinación de las alineaciones, o bien la aplicación de lo que denominamos "ternas pitagóricas" (Fig. 3).

En planimetrías en las que se ha comprobado que lo que se refleja es el norte geográfico presentamos algunos ejemplos de la aplicación de estas "ternas pitagóricas": como en la alineación de Alejandría (a latitud $31^{\circ}$ ), citada con anterioridad y ejemplo de ciudad simbólica (Haselberger 1999: 93; Ferro y Magli 2012: 386), equivalente a la terna 5:12:13. En el caso de las planimetrías de Barcino o de la parcelación en torno a Ebora, su orientación parece coincidir con la aplicación de la terna 3:4:5 (Orfila et alii 2014d: 106). En Thugga (Túnez), el templo B se orienta siguiendo la ratio $3: 5$, en lectura de terna $3: 4: 5$, mientras que la casa de Trifolium tiene una ratio 5:12, correspondiente a la terna 5:12:13 (Orfila et alii 2014d: 105, figs. 49 y 48). También se ha reconocido sobre parcelarios de la Narbonense (Pérez 1995; Assénat 2006), Gran Bretaña (Ferrar y Richardson 2003), Túnez (Decramer y Hilton 1996), o en los de la antigua colonia de Arausio (Orange) (Decramer et alii 2003 y 2006).

El uso de la varatio también se ha deducido a partir del análisis sobre planimetrías publicadas. Welfare y Swan (1995) presentaron las orientaciones de diversos campamentos romanos de Inglaterra, afirmando que algunos de ellos compartían una misma orientación, pero que esta se debía al relieve de la zona y a la existencia de vías cercanas a los mismos. Richardson hizo una revisión de 67 orientaciones, determinando que el desvío con respecto al norte se relacionaba con el uso de triángulos de ángulo recto, en los que los valores de los catetos fueron en unidades, es decir, en fracciones, como 2/3, 2/5, 3/4, etc. (Richardson 2005: 418). Este autor encontró relaciones parecidas en otras 

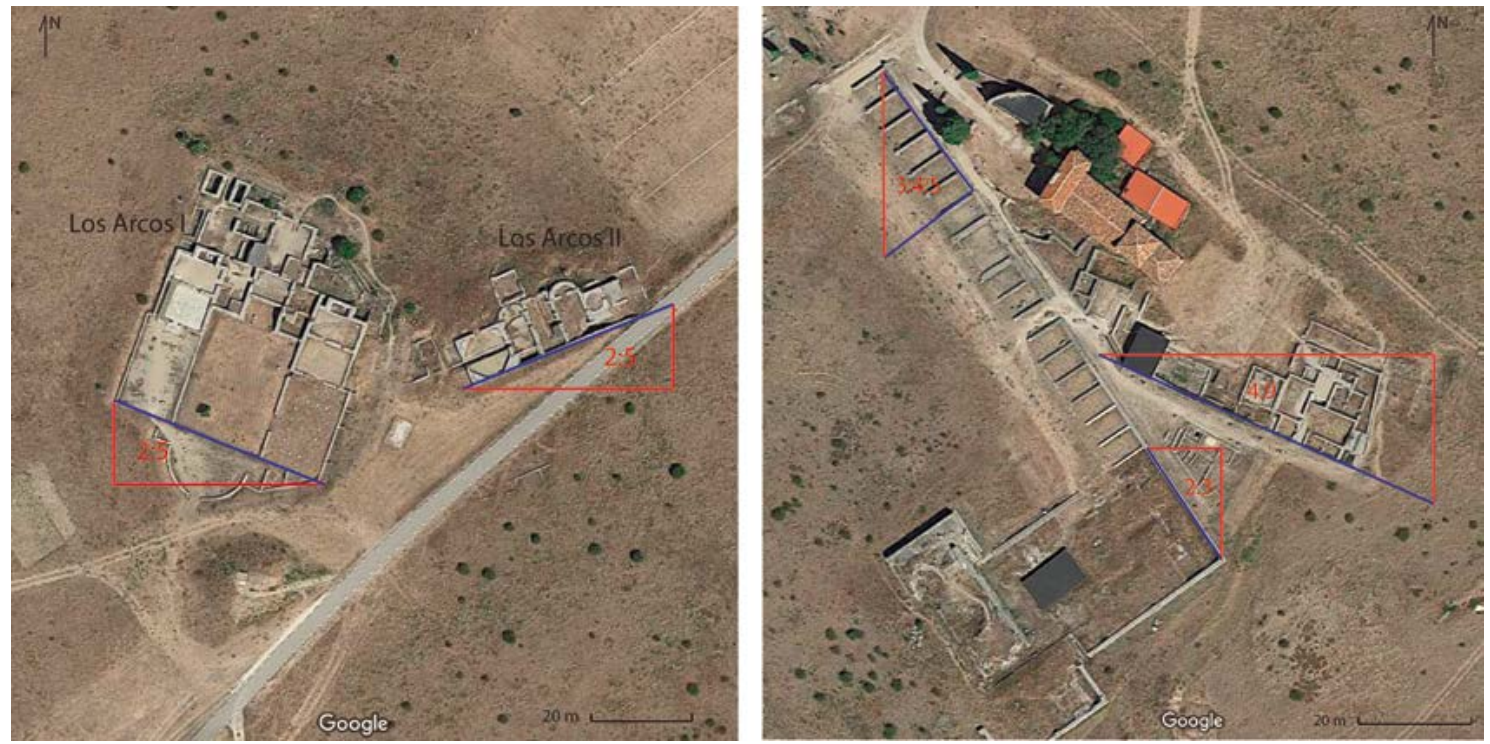

Figura 3. Aplicación de la varatio y su variante con ternas pitagóricas en Clunia (elaboración propia).

zonas, como en España, en los campamentos de Renieblas IV (a $8^{\circ}$, ratio $\left.1 / 3\right)$; Renieblas v (a $22^{\circ}$, ratio $2 / 5$ ), etc. (Richardson 2005: 420) ${ }^{13}$. Por otro lado, un estudio posterior de los lugares estudiados por Richardson en Gran Bretaña que considera también el horizonte circundante a cada lugar, apunta a una posible intencionalidad astronómica en su orientación relacionada con ortos y ocasos solares en fechas relevantes del calendario militar romano; como el 1 de marzo (Rodríguez-Antón et alii 2016b).

En relación con estos resultados y los de otros publicados con posterioridad para ciudades en diferentes regiones de lo que fue el imperio romano (Magli 2008; González-García y Magli 2015; Rodríguez-Antón et alii 2016a; Rodríguez-Antón et alii 2018a), basados en el estudio estadístico de un corpus relativamente completo de orientaciones, se concluyó el carácter solar y no aleatorio de estas. Por ejemplo, Giulio Magli, apoyándose en un listado de 38 ciudades en Italia, identificó 5 que tenían una divergencia referente al norte que oscilaba entre $36^{\circ}$ y $37^{\circ}$, una variación muy cercana a los $36^{\circ} 30^{\prime}$, correspondientes a la ratio 3:4 propuesta por nosotros usando ternas pitagóricas. Con su eje girado hacia el NE cita a Ariminum (Rímini) a $37^{\circ} \mathrm{y}$

${ }^{13}$ Como ya se ha comentado, este planteamiento fue rebatido por Peterson (2007: 106) que consideró que las coincidencias de orientaciones entre los campamentos estudiados no serían debidas a la aplicación de una misma técnica y con unos mismos criterios, si no al azar. Sin embargo, basándonos en resultados publicados con posterioridad (ver referencias en el texto), hoy podemos decir que probablemente estas orientaciones no sean aleatorias y que pueden vincularse al uso de la varatio.
Verona a $36^{\circ}$; hacia el SE, Augusta Bagiennorum (Bene Vagienna) a $36^{\circ}$, y Alba Fucens (Massa d'Albe) y Grumentum (Grumento Nova) a $37^{\circ}$; un poco más alejadas señala a Cosa y Luni a $38^{\circ}$ al SE (Magli 2008: 67, tabla I). Otro ejemplo lo constituye el santuario de la ciudad de Nertobriga (Berrocal-Rangel et alii 2014), cuyas orientaciones, presentadas en grados, tienen sus correspondencias en ratios de varatio o fracciones, según se quiera denominar (Orfila et alii 2017b). Así $4^{\circ} 05^{\prime}$ equivale a la $1: 12$; mientras que $14^{\circ}$ lo es a $1: 4$; en el caso de $104^{\circ}$ su valor es $1: 4$, y así sucesivamente en $28^{\circ} 60^{\prime}$ que es 6:11, o 122 50 ' grados a 7:11.

Incluimos aquí una tabla (Fig. 4) en la que se recogen comprobaciones que se han llevado a cabo in situ, utilizando el instrumental y procedimiento descritos en las fuentes literarias, combinando el gnomon con la groma, y recogiendo los datos obtenidos mediante Estación Total para luego volcarlos sobre planimetría con el programa Autocad. En todos los casos se han identificado desviaciones en relación con la línea meridiana que responden bien a alguna ratio de la varatio o bien a alguna "terna pitagórica".

Junto a estas comprobaciones, de forma paralela y en el marco de los Proyectos OAS III y IV del Plan Nacional de Astronomía y Astrofísica (Belmonte et alii 2016), se han realizado una serie de pruebas sobre diferentes ciudades hispano-romanas, en este caso, comprobando las orientaciones in situ con instrumentos modernos. Hasta la fecha se cuenta con una muestra de 81 medidas de orientaciones de ciudades de distinto estatus y de campamentos romanos (Rodríguez-Antón et alii 2018). En ellas se ha medido el 


\begin{tabular}{|c|c|c|c|c|c|}
\hline Yacimiento & $\begin{array}{l}\text { Ubicación del } \\
\text { gnomon }\end{array}$ & $\begin{array}{l}\text { Varatio o } \\
\text { ternas }\end{array}$ & Fracción & $\begin{array}{c}\text { Acimut } \\
\text { equivalente }\end{array}$ & Bibliografía \\
\hline $\begin{array}{l}\text { Clunia (Peñalba de } \\
\text { Castro, Burgos) }\end{array}$ & $\begin{array}{l}\text { Foro, junto a la } \\
\text { basílica }\end{array}$ & $\begin{array}{l}\text { TERNAS/ } \\
\text { VARATIO }\end{array}$ & $3: 4: 5 / 2: 3^{1}$ & $37^{\circ} / 34^{\circ}$ & $\begin{array}{l}\text { (Sánchez y Marín } \\
\text { 2014, 163) / } \\
\text { INÉDITO }\end{array}$ \\
\hline $\begin{array}{l}\text { Clunia (Peñalba de } \\
\text { Castro, Burgos) }\end{array}$ & Termas, Arcos 1 & VARATIO & $2: 5$ & $22^{\circ}$ & INÉDITO \\
\hline $\begin{array}{l}\text { Clunia (Peñalba de } \\
\text { Castro, Burgos) }\end{array}$ & Termas, Arcos 2 & VARATIO & $2: 5$ & $22^{\circ}$ & INÉDITO \\
\hline $\begin{array}{l}\text { Clunia (Peñalba de } \\
\text { Castro, Burgos) }\end{array}$ & Casa 3 & VARATIO & $4: 9$ & $66^{\circ}$ & INÉDITO \\
\hline $\begin{array}{l}\text { Torreparedones } \\
\text { (Baena, Córdoba) }\end{array}$ & $\begin{array}{l}\text { zona al oeste del } \\
\text { macellum }\end{array}$ & $\begin{array}{l}\text { NORTE } \\
\text { SOLAR }\end{array}$ & -- & & $\begin{array}{c}\text { (Sánchez y Marín } \\
\text { 2014, 180) }\end{array}$ \\
\hline $\begin{array}{l}\text { Puente Tablas (ibérico) } \\
\text { (Jaén) }\end{array}$ & $\begin{array}{l}\text { zona central de la } \\
\text { estructura urbana } \\
\text { modulada }\end{array}$ & TERNAS & $5: 12: 13$ & $22^{\circ}$ & $\begin{array}{c}\text { (Sánchez y Marín } \\
\text { 2014, 186) }\end{array}$ \\
\hline $\begin{array}{l}\text { Pollentia (Alcudia, } \\
\text { Mallorca) }\end{array}$ & $\begin{array}{l}\text { Foro, frente al } \\
\text { capitolio y al norte } \\
\text { del edículo }\end{array}$ & VARATIO & $3: 5^{2}$ & $33^{\circ}$ & $\begin{array}{c}\text { (Sánchez y Marín } \\
\text { 2014, 183) }\end{array}$ \\
\hline $\begin{array}{l}\text { Sanisera (Sa Nitja, } \\
\text { Menorca) }\end{array}$ & $\begin{array}{l}\text { sobre estructuras } \\
\text { tardías }\end{array}$ & TERNAS & $3: 4: 5$ & $53^{\circ}$ & $\begin{array}{c}\text { (Sánchez y Marín } \\
\text { 2014, 167) }\end{array}$ \\
\hline $\begin{array}{l}\text { Valeria (Las Valeras, } \\
\text { Cuenca) }\end{array}$ & Foro & TERNAS & $\begin{array}{l}\text { Combinación de } \\
\text { 3:4:5 y 5:13:13 }\end{array}$ & $60^{\circ}$ & $\begin{array}{c}\text { (Sánchez y Marín } \\
\text { 2014, 171) }\end{array}$ \\
\hline $\begin{array}{l}\text { Italica-Nova Urbs- } \\
\text { (Santiponce, Sevilla) }\end{array}$ & Casa del Planetario & TERNAS & $\begin{array}{l}\text { Combinación de } \\
\text { 3:4:5 y } 5: 13: 13\end{array}$ & $31^{\circ}$ & $\begin{array}{c}\text { (Sánchez y Marín } \\
\text { 2014, 176) }\end{array}$ \\
\hline $\begin{array}{l}\text { Italica-Vetus Urbs- } \\
\text { (Santiponce, Sevilla) }\end{array}$ & Termas Menores & TERNAS & $5: 12: 13$ & $66^{\circ}$ & $\begin{array}{c}\text { (Sánchez y Marín } \\
\text { 2014, 177) }\end{array}$ \\
\hline $\begin{array}{l}\text { Baelo Claudia (Tarifa, } \\
\text { Cádiz) }\end{array}$ & Foro & VARATIO & $1: 3$ & $72^{\circ}$ & INÉDITO \\
\hline Ammaia (Portugal) & Puerta Sur & VARATIO & $9: 10$ & $43^{\circ}$ & INÉDITO \\
\hline Ammaia (Portugal) & Puerta Sur & VARATIO & $9: 10$ & $42^{\circ}$ & INÉDITO \\
\hline
\end{tabular}

Figura 4. Resultados obtenidos a través de instrumental recreado de época romana (elaboración propia). 1 En la medición llevada a cabo en esta ciudad en el año 2010, manualmente a través del traslado por triangulación de la línea de la pared chequeada sobre la línea norte obtenida mediante un gnomon de obra, se apercibió una relación a través de "ternas pitagóricas" 3:4:5. Posteriormente, en el chequeo llevado a cabo en septiembre de 2015 pudo comprobarse, utilizando Estación Total, que se podía contabilizar el desvío en relación al norte, mediante la varatio fracción 2:3. 2 En el test llevado a cabo sobre imagen de Google Earth (Equipo sotoer 2014: 79) se apreció una relación DE VARATIO DE 3:5.

acimut de la trama urbana, bien a partir de los restos de esta en las vías y calles o a partir de los ejes de las plazas públicas (fora) o de otras estructuras edilicias. En aquellos casos en los que se cuenta con más de una medida, bien por un cambio de orientación tras una ampliación/refundación (como puede ser el caso de Itálica), bien por la existencia de un foro y una trama con orientaciones no coincidentes, se consideran ambas.

Las medidas se han realizado con una brújula de precisión, corrigiendo la declinación magnética en cada caso particular basándonos en un modelo de magnetismo terrestre reciente: World Magnetic Model $(\mathrm{WMM})^{14}$. En cada yacimiento se realizaron varias medidas con más de un instrumento con el objetivo de obtener valores más aproximados a los reales, de modo que lo que se representa son los valores medios obtenidos en cada lugar. Esto es necesario dado el deterioro, en mayor o menor grado, de los restos arqueológicos con el paso del tiempo, además de por la

\footnotetext{
${ }^{14}$ Magnetic Field Calculators del National Centers for Environmental information (NOAA): https://www.ngdc.noaa.gov/ geomag/WMM/
} 
irregularidad intrínseca de las propias estructuras derivadas de las limitaciones técnicas de la época. Una medida individual con la brújula de precisión tiene un error nominal de $\pm 14^{\circ}$. El error medio en la declinación magnética calculada por el NOAA (National Oceanic and Atmosferic Administration) a partir del WMM (World Magnetic Model) es $\pm 1 / 2^{\circ}$, de modo que se establece un error de $\pm 1 / 2^{\circ}$ para el acimut. Hay factores, como el estado de conservación de los restos, que hacen que podamos considerar mayor incertidumbre en algunos lugares, pero para el análisis inicial de la muestra consideramos el mismo error en todos los casos, siendo conscientes de la necesidad de ajustar estos errores en sitios particulares. En la Figura 5 se muestra el mapa con los lugares cuyas orientaciones se han incluido en este trabajo.

Respecto a la varatio, en primer lugar, se han calculado los valores de los ángulos de proporciones hasta 12:12, evitando repetir ángulos iguales originados por lados en igual relación de proporcionalidad -como en el caso de 1:3, 2:6, etc. en el que solo se ha tenido en cuenta el 1:3- como ya se ha indicado previamente.
La idea de usar valores hasta 12:12 viene motivada porque los triángulos pitagóricos más sencillos son el 3:4:5 y el 5:12:13, marcando este último un límite superior a los valores de varatio considerados en este estudio. Se ha establecido este límite a partir del uso de la terna 5:12:13 en la arquitectura romana (Roskams 2001: 96; Orfila 2014b: 67) y porque, a falta de otro criterio reflejado en las fuentes, la elección de otro valor habría sido puramente subjetiva. No obstante, a la hora de discernir la aplicación de una relación de varatio en general se considerará más plausible aquella que involucre números menores, por ser de más fácil manejo. De este modo se recrean los posibles ángulos que subtendería una ciudad si se aplicase una determinada varatio colocando los catetos del triángulo sobre los ejes cardinales (Fig. 2A: 3a, 3b). Para su obtención se ha procedido calculando la arcotangente para cada par de catetos, llamémoslos $\mathrm{x}, \mathrm{y}$, de los triángulos involucrados.

De esta forma el ángulo de orientación sería $\alpha$ o bien, su simétrico, $360^{\circ}-\alpha$. La orientación escogida para la trama urbana sería la de la hipotenusa y su perpendicular, trazada mediante el uso de la groma o la bisectriz.

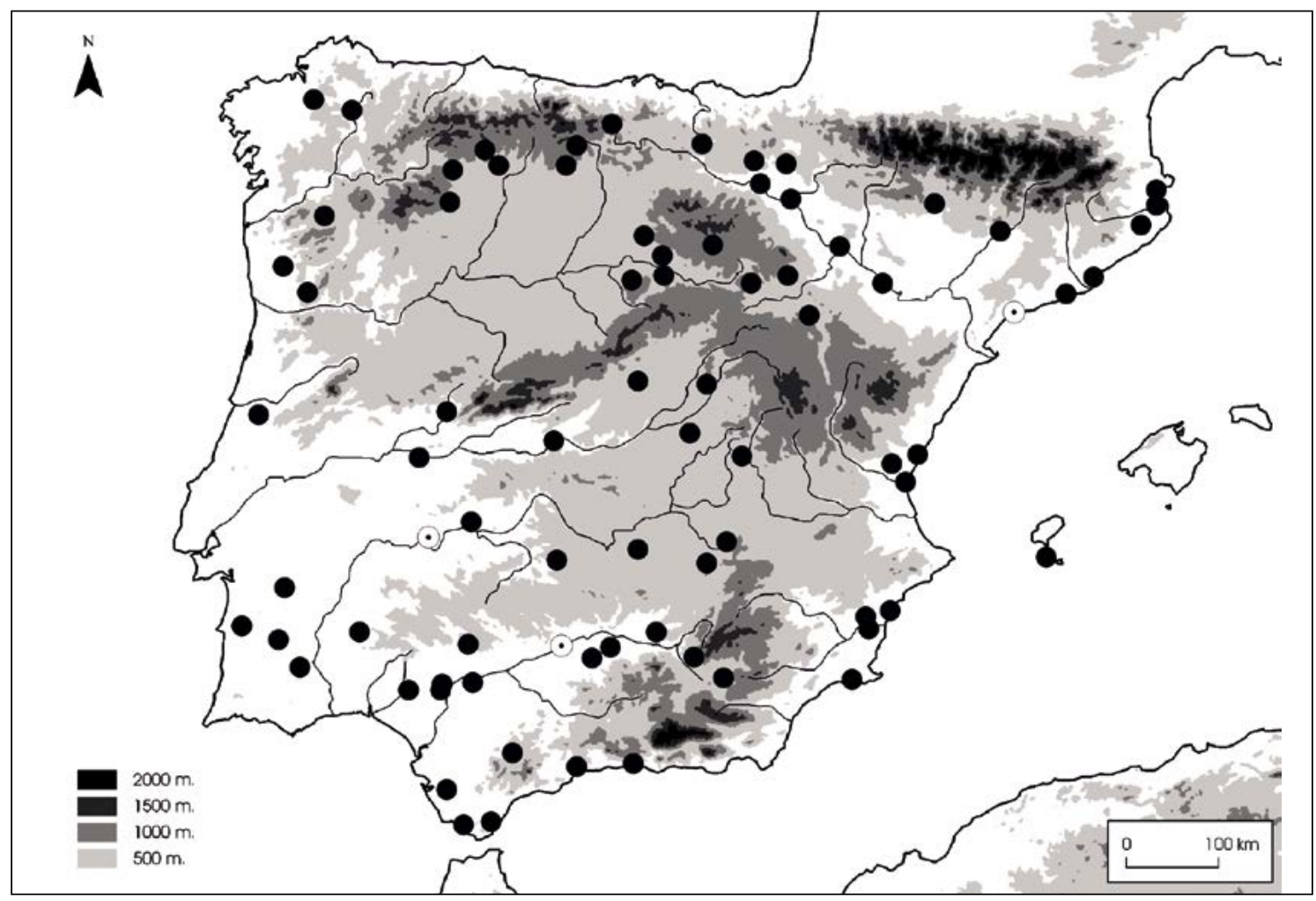

Figura 5. Asentamientos romanos cuyas orientaciones se incluyen en la muestra estudiada. Estos datos han sido tomados con una brújula de precisión y corregidos de declinación magnética (ver texto) (elaboración propia). 
La Figura 6 muestra la gama de orientaciones posibles resultantes de esta primera forma de aplicación de la varatio. Los resultados están expresados para los cuatro ejes del entramado urbano, y dada la simetría del proceso, observamos que se repiten los patrones en cada sector de circunferencia delimitado por los puntos cardinales.

Para la representación de los datos se han usado curvigramas frente a los tradicionales histogramas de cajas. En este caso lo que se representa son funciones continuas que suavizan el histograma de cajas al multiplicar los valores de la muestra por la función de densidad llamada kernel, de utilidad cuando la distribución de datos no sigue una conocida (gaussiana, exponencial, etc.). El kernel define la forma de los máximos y se le asigna un ancho de banda que influye en el aspecto de la distribución resultante al afectar a la dispersión de probabilidad en torno a un punto. En este caso se ha usado un kernel de Epanechnikov, pues en el gaussiano los máximos son más anchos y afectaría a su significancia, y en la Figura 6 se ha escogido un ancho de banda de $1^{\circ}$. El ancho de banda escogido se adapta al tamaño de la muestra, a la densidad de datos y al error, de modo que el curvigrama resultante se puedan apreciar con claridad los máximos principales.

Así, en la Figura 7 se observa esta misma distribución restringida a un sector entre los ángulos $\left[45^{\circ}\right.$, $135^{\circ}$ ]. La motivación de escoger tal división es que, siguiendo una definición puramente geométrica, podríamos considerar como un decumano la calle que transcurre dentro de este sector de acimut, mientras que si tiene un ángulo menor a $45^{\circ}$ o mayor que $135^{\circ}$ pasaría a ser un cardo. Es, por tanto, una de las divisiones simétricas posibles del diagrama de la Figura 6. En la Figura 7 se incluye también la distribución de acimuts de los asentamientos romanos, para lo que se ha usado un ancho de banda de $1^{\circ}$ escogiendo el doble del error de acimut y atendiendo al tamaño de la muestra. Esta última distribución es exactamente igual en los tres sectores restantes de la circunferencia debido a la ortogonalidad de las tramas urbanas estudiadas. Los valores de los máximos de acimut indicados en la Figura 7 se muestran en la tabla de la Figura 8 con números romanos. Los valores de las orientaciones de cada lugar y las varationes que mejor se ajustan a estos se pueden consultar en la tabla de la Figura 9.

Con el fin de comparar si una dirección en la práctica es compatible con los métodos geométricos propuestos, se ha considerado un valor conservador de $\pm 2^{\circ}$, derivado de los propios errores de las medidas de acimut $\left( \pm 1 / 2^{\circ}\right)$ y de los derivados de la aplicación de la varatio, que hemos considerado $1,5^{\circ}$ como aproximación en este trabajo.

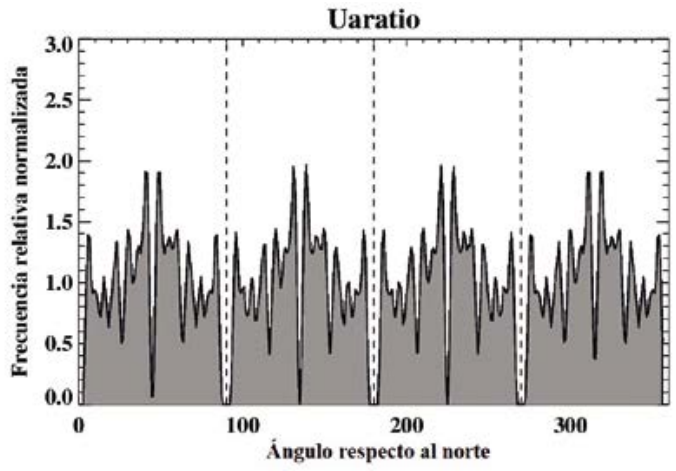

Figura 6. Distribución de ángulos de varatio desde 1:2 a 11:12. Solo se incluyen una vez las relaciones de proporcionalidad equivalentes puesto que devuelven el mismo ángulo; esto es, se considera $1: 3$ y no $2: 6$ o 4:12 y lo mismo para el resto de fracciones que guardan equivalencia entre sí (elaboración propia).

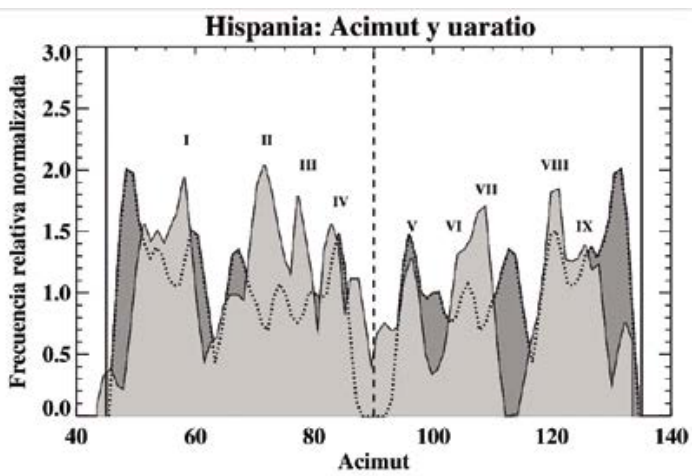

Figura 7. Histograma de acimut de la muestra de orientaciones estudiada (gris claro) frente a la distribución de los mismos ángulos de varatio de la figura anterior (gris oscuro) para un sector de $90^{\circ}$ de amplitud, correspondiente a la fracción de horizonte en la que consideramos que se encontraría un decumano y centrada en el punto cardinal este (línea discontinua) (elaboración propia).

A primera vista, aunque existen máximos de ambas distribuciones que coinciden con mínimos y viceversa, lo realmente interesante es la aparente coincidencia de los mínimos principales de varatio con mínimos de acimut de la muestra, lo que indica una menor presencia de orientaciones hacia ángulos que no se corresponden con una varatio. Estos mínimos absolutos coincidentes se encuentran en los puntos cardinales (aunque Basti y Gerunda tienen orientación cardinal) y en los inter-cardinales $\left(45^{\circ}, 135^{\circ}, 225^{\circ}\right.$ y $315^{\circ}$ ); estos últimos actúan como límites de cada sector de acimut de anchura de $90^{\circ}$ y sus valores coinciden con la varatio 1:1. Otro mínimo relativo coinci- 
138 ORFILA PONS, RODRÍGUEZ-ANTÓN, CHÁVEZ-ÁLVAREZ, GONZÁLEZ-GARCÍA, SÁNCHEZ LÓPEZ, BELMONTE

\begin{tabular}{|c|c|c|c|}
\hline \multirow{2}{*}{ Máximo } & \multirow{2}{*}{ Acimut $\left(^{\circ}\right)$} & \multicolumn{2}{|c|}{ Varatio } \\
\hline & & $\left({ }^{\circ}\right)$ & X:Y \\
\hline \multirow{5}{*}{ I } & \multirow{5}{*}{58} & 51,34 & $5: 4$ \\
\hline & & 57,5 & $11: 7$ \\
\hline & & 58,0 & $8: 5$ \\
\hline & & 59,0 & $5: 3$ \\
\hline & & 59,7 & $12: 7$ \\
\hline II & 72 & $71 \frac{1}{2}$ & $3: 1$ \\
\hline \multirow{2}{*}{ III } & \multirow{2}{*}{$771 / 2$} & $771 / 2$ & $9: 2$ \\
\hline & & 78,8 & $5: 1$ \\
\hline IV & 83 & 83,6 & $9: 1$ \\
\hline \multirow{4}{*}{$\mathbf{V}$} & \multirow{4}{*}{$961 / 2$} & 95,2 & $1: 11$ \\
\hline & & 95,7 & $1: 10$ \\
\hline & & 96,3 & $1: 9$ \\
\hline & & 97,1 & $1: 8$ \\
\hline VI & $1041 / 2$ & 104,0 & $1: 4$ \\
\hline VII & $108^{1 / 2}$ & 108,4 & $1: 3$ \\
\hline \multirow{4}{*}{ VIII } & \multirow{4}{*}{$1191 / 2$} & \multirow{2}{*}{119,0} & $4: 7$ \\
\hline & & & $5: 9$ \\
\hline & & 120,2 & $7: 12$ \\
\hline & & 120,9 & $3: 5$ \\
\hline IX & 125 & \multicolumn{2}{|c|}{$5: 7\left(125,5^{\circ}\right)$} \\
\hline
\end{tabular}

Figura 8. Máximos correspondientes al histograma de acimut en un sector de $90^{\circ}$ de amplitud, indicados en la Figura 7 en la que se incluyen los ángulos correspondientes a cada máximo y las varationes que más se ajustan a esos valores (elaboración propia).

\begin{tabular}{|c|c|c|c|}
\hline Yacimiento & $\mathbf{A}\left(\left(^{\mathbf{o}}\right)\right.$ & $\Phi\left(^{\circ}\right)$ & Varatio/Terna \\
\hline Cidadela (T) & 107 & 43,10 & $\mathrm{VII} / 7: 24: 25$ \\
\hline Lucus Augusti (T) & 71 & 43,00 & II \\
\hline Juliobriga (T) & $1241 / 4$ & 42,99 & IX/3:4:5 \\
\hline Iruña-Veleia & $1081 / 4$ & 42,83 & VII \\
\hline M. Cildá (T) & $701 / 2$ & 42,75 & II \\
\hline Pisoraca (T) & $116^{1 / 2}$ & 42,59 & 1:2/VIII \\
\hline Legio (T) & $693 / 4$ & 42,60 & 8:3/II/5:12:13 \\
\hline Santa Cris (T) & 104 & 42,54 & $\mathrm{VI} / 7: 24: 25$ \\
\hline Lancia (T) & $1193 / 4$ & 42,50 & VIII \\
\hline Asturica (T) & $701 / 2$ & 42,46 & II \\
\hline Santacara (T) & $51^{1 / 4}$ & 42,36 & $9: 7$ \\
\hline Arcobriga (T) & $513 / 4$ & 42,29 & $9: 7$ \\
\hline Los Bañales (T) & $501 / 4$ & 42,28 & $6: 5 / 11: 9$ \\
\hline
\end{tabular}

\begin{tabular}{|c|c|c|c|}
\hline Yacimiento & $\mathbf{A}\left({ }^{\circ}\right)$ & $\Phi\left(\left(^{\circ}\right)\right.$ & Varatio/Terna \\
\hline Roses (T) & $104 \frac{1}{2}$ & 42,25 & $\mathrm{VI} / 7: 24: 25$ \\
\hline Emporion (T) & $661 / 4$ & 42,13 & 7:3/9:4/5:12:13 \\
\hline Labitolosa (T) & $491 / 2$ & 42,13 & $7: 6$ \\
\hline Petavonium (T) & $1201 / 2$ & 42,08 & VIII \\
\hline Aquis Q. (T) & $1281 / 2$ & 42,00 & IX/4:5/3:4:5 \\
\hline Gerunda (T) & $913 / 4$ & 41,98 & $\mathrm{C}$ \\
\hline Numantia (T) & $1093 / 4$ & 41,80 & VII \\
\hline Ieso $(T)$ & $781 / 4$ & 41,78 & III/9:40:41 \\
\hline Clunia (T) & $551 / 2$ & 41,76 & $2: 3 / 3: 4: 5$ \\
\hline Cesaraugusta (T) & $1241 / 4$ & 41,65 & IX \\
\hline Uxama (T) & $863 / 4$ & 41,58 & $12: 1$ \\
\hline Iluro (T) & 65 & 41,54 & $11: 5 / 5: 12: 13$ \\
\hline Bracara $(\mathbf{T})$ & $721 / 4$ & 41,50 & II \\
\hline $\operatorname{Barcino}(\mathbf{T})$ & $126^{3} / 4$ & 41,38 & IX/3:4:5 \\
\hline Andelos (T) & 118 & 41,38 & VIII \\
\hline Bilbilis (T) & 76 & 41,36 & III \\
\hline Celsa (cardo i) (T) & $781 / 4$ & 41,36 & III/9:40:41 \\
\hline Tiermes (T) & 78 & 41,33 & III/9:40:41 \\
\hline Confluentia (T) & $543 / 4$ & 41,29 & $7: 5 / 3: 4: 5$ \\
\hline Tongobriga (T) & 73 & 41,16 & II/7:24:25 \\
\hline \multirow{2}{*}{$\operatorname{Tarraco}(\mathbf{T})$} & 124 & 41,11 & IX/3:4:5 \\
\hline & $1191 / 2$ & 41,11 & VIII \\
\hline La Caridad (T) & $961 / 4$ & 40,83 & $\mathrm{~V}$ \\
\hline Complutum (T) & $681 / 2$ & 40,50 & $5: 2 / 5: 12: 13$ \\
\hline Ercavica (T) & $801 / 2$ & 40,40 & $6: 1$ \\
\hline Caparra (L) & $541 / 2$ & 40,16 & $7: 5 / 3: 4: 5$ \\
\hline Conimbriga $(\mathrm{L})$ & $571 / 2$ & 40,08 & I \\
\hline Cesarobriga(L) & 95 & 39,95 & $1: 12$ \\
\hline Segobriga $(T)$ & $753 / 4$ & 39,88 & 4:1/III?/ 7:24:25 \\
\hline Valeria (T) & $120^{3 / 4}$ & 39,80 & VIII/7:12 \\
\hline \multirow{2}{*}{ Saguntvm (T) } & $1091 / 4$ & 39,67 & VII \\
\hline & $831 / 2$ & 39,67 & IV \\
\hline Lauro (T) & 67 & 39,62 & $12: 5 / 5: 12: 13$ \\
\hline Valentia (T) & $971 / 4$ & 39,50 & $\mathrm{~V}$ \\
\hline Cáceres V (L) & 107 & 39,50 & VII/7:24:25 \\
\hline Libisosa (T) & $871 / 4$ & 38,95 & $12: 1\left(\approx 85^{\circ}\right)$ \\
\hline Metellinum (L) & 121 & 38,95 & VIII (3:5) \\
\hline Emerita (L) & $523 / 4$ & 38,90 & $9: 7 / 3: 4: 5$ \\
\hline Mentesa (T) & 53 & 38,70 & $4: 3 / 11: 8 / 3: 4: 5$ \\
\hline
\end{tabular}




\begin{tabular}{|c|c|c|c|}
\hline Yacimiento & $\mathbf{A}\left({ }^{\mathbf{o}}\right)$ & $\Phi\left(^{\circ}\right)$ & Varatio/Terna \\
\hline Formentera $(\mathbf{T})$ & $1221 / 4$ & 38,68 & 5:8/7:11 \\
\hline Oretum (T) & $871 / 4$ & 38,67 & 12:1/11:1/10:1 \\
\hline Sisapo (T) & $563 / 4$ & 38,65 & I \\
\hline L.Iulia (L) & 58 & 38,57 & I \\
\hline Lucentum (T) & $1251 / 4$ & 38,30 & IX/3:4:5 \\
\hline \multirow{2}{*}{ Ilici (T) } & $821 / 2$ & 38,25 & IV \\
\hline & 78 & 38,25 & III/9:40:41 \\
\hline Mirobriga (L) & $451 / 2$ & 38.00 & 1:1 \\
\hline Pax Iulia (L) & $1271 / 2$ & 38.00 & $7: 9 / 3: 4: 5$ \\
\hline Cástulo (B) & $733 / 4$ & 38.00 & II/7:24:25 \\
\hline Turobriga (B) & $963 / 4$ & 37,94 & V \\
\hline Corduba(río) & 59 & 37,88 & I \\
\hline Corduba(templo) & $933 / 4$ & 37,88 & $1: 12$ \\
\hline Porcuna (B) & $631 / 2$ & 37,87 & $2: 1$ \\
\hline Torreparedones(B) & 83 & 37,75 & IV \\
\hline Munigua (B) & $591 / 2$ & 37,71 & I \\
\hline Myrtilis (L) & $843 / 4$ & 37,65 & IV \\
\hline Carthago Nova (T) & $61 \frac{1 / 2}{2}$ & 37,62 & $11: 6$ \\
\hline Basti (B) & $901 / 2$ & 37,51 & $\mathrm{C}$ \\
\hline Carmona (B) & $1031 / 4$ & 37,47 & VI \\
\hline Italica I (B) & $573 / 4$ & 37,40 & I \\
\hline Italica R (B) & 72 & 37,40 & II/7:24:25 \\
\hline Acinipo (B) & $821 / 4$ & 36,80 & IV \\
\hline Sexi (B) & $1321 / 2$ & 36,73 & 10:11/11:12 \\
\hline Malaca (B) & $105^{1 / 2}$ & 36,72 & $\mathrm{VI} / 7: 24: 25$ \\
\hline Medina Sidonia (B) & $733 / 4$ & 36,45 & II/7:24:25 \\
\hline Carteia (B) & $132^{1 / 4}$ & 36,12 & 10:11/11:12 \\
\hline Baelo (B) & 108 & 36,08 & $\mathrm{VII} / 7: 24: 25$ \\
\hline
\end{tabular}

sencillas; por ejemplo el II con 1:3 o el VIII con 3:5 como una de sus posibilidades.

Como ya se ha mencionado en secciones anteriores, existe una simetría dentro de un sector de $90^{\circ}$ de anchura como el escogido para representar la Figura 7, de manera que los ángulos tanto de las orientaciones de las ciudades como de las varationes pueden restringirse a un sector de $45^{\circ}$ de amplitud. De este modo, al considerar el sector $\left[0^{\circ}, 45^{\circ}\right]$ se estaría atendiendo a las orientaciones iguales pero opuestas respecto a la línea norte-sur. Así, en este caso se tienen en cuenta simultáneamente ratios que involucran lados iguales independientemente de su configuración a lo largo de los ejes cardinales -por ejemplo, las varationes 1:3 y 3:1 se consideran como una única: la 1:3-. Como ya se dijo en la Sección 4, mediante este ejercicio se limita el número de posibles relaciones de proporcionalidad, lo que facilita su asociación con los acimuts de las ciudades. El procedimiento para restringir los datos al sector $\left[0^{\circ}, 45^{\circ}\right]$ consiste en no modificar aquellos acimuts con valores dentro de este rango y, para los que coinciden con ángulos comprendidos entre $\left[45^{\circ}, 90^{\circ}\right]$, se toma el ángulo subtendido respecto a $45^{\circ}$ que llamamos $\Phi$. Este se tiene en cuenta para el cálculo del nuevo ángulo respecto al norte, que denominamos $\alpha$. De este modo, los ángulos que antes se orientaban al oeste de la línea meridiana son ahora $\alpha=45^{\circ}-\Phi$. El resultado de este cálculo se representa en la Figura 10 , y los valores de los máximos que en esta aparecen están recogidos en la tabla de la Figura 11. El ancho de banda escogido para representar estos histogramas es de $34^{\circ}$, al aumentar en número de elementos de la muestra disponibles por sector. De este modo es posible apreciar los patrones de orientación existentes con menor dispersión, sin que la distribución adquiera un aspecto demasiado suavizado (manteniendo un valor de $1^{\circ}$ ), o demasiado "dentado" si se escogiese un valor muy pequeño. Al tener un rango de acimut más pequeño con el mismo número de medidas, conviene reducir el criterio por el que se aproxima un acimut a una relación de varatio, estableciendo en este caso un margen de $\pm 1^{\circ}$.

Por simetría, si se compara este histograma (Fig. 10) con el de la Figura 7, se extrae que el máximo $\mathrm{x}$ contiene las medidas que aparecen en los picos IV y v de la Figura 7 y, en la misma línea, el xi aglutina el III y el VI, el XII los II y VII y el XIII los máximos i y viII (ver valores en las tablas de las Figs. 8 y 11). El siguiente paso ha sido comprobar la presencia de ciudades cuyas orientaciones se ajustaran al empleo de ternas pitagóricas, otra de las hipótesis principales planteadas en el presente artículo (Fig. 2A: 2a, 2b). 


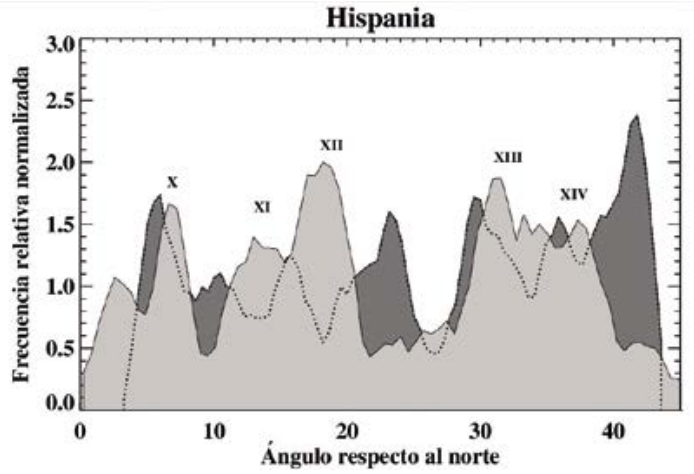

Figura 10. Histograma de acimut de la muestra de orientaciones estudiada (gris claro) y distribución de los mismos ángulos de varatio que en la figura anterior (línea negra) para un sector de $45^{\circ}$ de amplitud. Los valores de los máximos de acimut indicados con números romanos se muestran en la Figura 11 (elaboración propia).

\begin{tabular}{|c|c|c|c|c|}
\hline \multirow{2}{*}{ Máximo } & \multirow{2}{*}{$\underset{\left({ }^{\circ}\right)}{\operatorname{Acimut}}$} & \multicolumn{2}{|c|}{ Varatio } & \multirow{2}{*}{$\begin{array}{c}\text { Máximos } \\
\text { equivalentes }\end{array}$} \\
\hline & & $\left({ }^{\circ}\right)$ & $\mathrm{X}: \mathrm{Y}$ & \\
\hline \multirow{3}{*}{$\mathbf{X}$} & \multirow{3}{*}{7} & 6,3 & $1: 9$ & \multirow{3}{*}{ IV V } \\
\hline & & 8,1 & $1: 7$ & \\
\hline & & 7,1 & $1: 8$ & \\
\hline \multirow{2}{*}{ XI } & \multirow{2}{*}{12} & 11,3 & $1: 5$ & \multirow{2}{*}{$\begin{array}{l}\text { III } \\
\text { VI }\end{array}$} \\
\hline & & 12,5 & $2: 9$ & \\
\hline XII & 18 & 18,4 & $1: 3$ & II/VII \\
\hline \multirow{4}{*}{ XIII } & \multirow{4}{*}{31.5} & $301 / 2$ & $7: 12$ & \multirow{4}{*}{$\begin{array}{c}\text { I } \\
\text { VIII }\end{array}$} \\
\hline & & 30,9 & $3: 5$ & \\
\hline & & 32,0 & $5: 8$ & \\
\hline & & 32,5 & 7:11 & \\
\hline \multirow{4}{*}{ XIV } & \multirow{4}{*}{37} & 36,0 & $8: 11$ & \multirow{4}{*}{ IX } \\
\hline & & 36,9 & $3: 4$ & \\
\hline & & 38,5 & $4: 5$ & \\
\hline & & 37,9 & $7: 9$ & \\
\hline
\end{tabular}

Figura 11. Máximos correspondientes al histograma de acimut en un sector de $45^{\circ}$ de amplitud, indicados en la Figura 10 en la que se incluyen los ángulos correspondientes a cada máximo y las varationes que más se ajusta a esos valores (elaboración propia).

En la Figura 12 se aprecia la distribución de acimuts de la muestra y se indican los ángulos correspondientes a las ternas 3:4:5 y 5:12:13, las más bajas y utilizadas habitualmente en la antigüedad, tal como se ha indicado en las secciones previas (Gros 1976: 676; Roskam 2001: 96; Orfila 2014a: 67). Como ejercicio complementario, se ha tratado de detectar si las

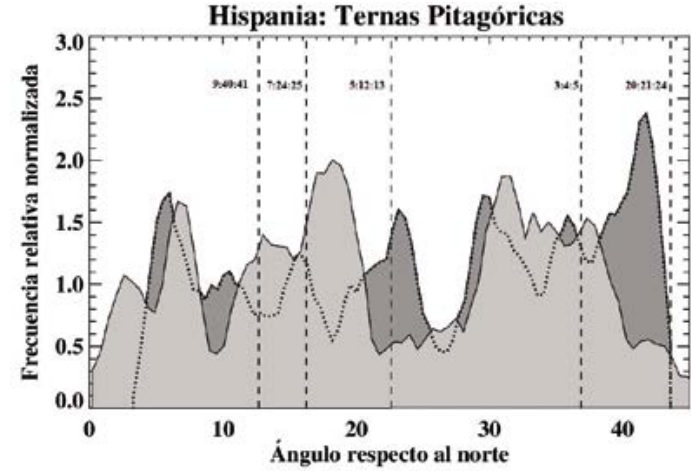

Figura 12. Histograma de acimut de la muestra de orientaciones estudiada (gris claro) y distribución de los mismos ángulos de varatio que en la figura anterior (gris oscuro) para un sector de $45^{\circ}$ de amplitud. Las líneas discontinuas verticales indican la hipotética orientación que tendría un yacimiento si estuviera orientado mediante el uso de las ternas pitagóricas 9:40:41 $\left(12,7^{\circ}\right), 7: 24: 25\left(16,25^{\circ}\right), 5: 12: 13\left(22,6^{\circ}\right), 3: 4: 5\left(36,8^{\circ}\right)$ y $20: 21: 29$ $\left(43,6^{\circ}\right)$ de la manera que se explica en el texto (elaboración propia).

orientaciones de la muestra se ajustan igualmente a ángulos correspondientes a ternas pitagóricas "mayores", como son la 9:40:41 $\left(12,7^{\circ}\right)$, la 7:24:25 $\left(16,25^{\circ}\right)$ y la 20:21:29 $\left(43,6^{\circ}\right)$. Si bien es lógico pensar que primaría la aplicación de variationes más sencillas, el hecho de introducirlas en la gráfica sirve como primera aproximación para corroborar o descartar un posible uso de estas relaciones.

Para responder a la pregunta de si la distribución de orientaciones de las ciudades romanas en Hispania podría obtenerse de los ángulos disponibles por el método de la varatio, hemos realizado la comparación de ambas distribuciones a través de un test de Kolmogorov-Smirnov (Baxter 2015). Este test comprueba si dos distribuciones de datos provienen de una misma población raíz. Esta es la hipótesis nula que vamos a comprobar. Si obtenemos valores de probabilidad muy bajos (en general definiremos nuestro criterio de rechazo con probabilidad menor a 0,05 , es decir, un nivel de confianza del $95 \%$ ), podremos rechazar la hipótesis nula y concluir que las orientaciones no se han obtenido mediante el método de la varatio. En este caso hemos realizado el test restringiéndonos a los rangos $\left[0^{\circ}, 90^{\circ}\right]$ y $\left[0^{\circ}, 45^{\circ}\right]$ en los que hemos considerado los mismos valores de acimut y varatio que en las Figuras 7 y 10, respectivamente. Los valores de la probabilidad resultantes son 0,5 para el rango $\left[0^{\circ}, 90^{\circ}\right]$ y 0,14 para $\left[0^{\circ}, 45^{\circ}\right]$. Ambos valores son mayores que 0,05 , luego no permiten descartar el uso de la varatio para la obtención de estas orientaciones; si bien tampoco afirma que ambas muestras tengan un mismo origen. 


\section{REFLEXIONES}

La frase de Vitruvio: "en la Arquitectura hay dos términos: lo significado y lo que significa. La cosa significada es aquella de la que uno se propone tratar; la significante es la demostración desarrollada mediante principios científicos" (Vitruvio I, I), resume, en cierta manera, los propósitos de este escrito.

En este caso focalizamos nuestros estudios en uno de los componentes de las construcciones: su orientación. En su elección tienen que ver diversidad de causas, tanto de tipo rituales como prácticas, hipotecadas ambas por el procedimiento que se aplicó para ejecutarlas. En este trabajo se han analizado un número considerable de alineaciones de estructuras romanas, tomadas in situ mediante dos procedimientos tal como se ha indicado: uno como podría haber sido el modo romano y el otro con metodología actual. La aplicación en paralelo de los dos sistemas de reconocimiento ha permitido comparar los resultados obtenidos, que han sido parejos.

Las comprobaciones in situ han puesto en evidencia, también, las dificultades a las que se enfrentaron los topógrafos, ingenieros y arquitectos de época romana, más teniendo en cuenta la operatividad del aparataje con el que se contaba -limitaciones técnicas y alcance de los aparatos-. En esos procesos, tal como Adam ya indicó, construcciones arquitectónicas, obras públicas, catastros rurales y urbanos, etc., fueron resultado de la aplicación de unas técnicas sistemáticas que tenían como preámbulo una operación topográfica (Adam 1982). Esa parte intermedia entre el plano dibujado por quien diseñó la obra y su plasmación sobre el terreno, es lo que se analiza en el procedimiento aplicado, dado que era en ese paso cuando se establecía la orientación elegida para la obra a ejecutar.

Los resultados sugieren que las orientaciones estudiadas no son aleatorias, pues aparecen una serie de máximos en las distribuciones que indican la existencia de patrones bien definidos. Se encuentran además unos mínimos absolutos que coindicen con valores que no se corresponden con un ángulo de varatio.

En un trabajo publicado recientemente, se han hallado interesantes similitudes entre los ángulos de varatio más frecuentes en Hispania con acimuts del sol en días señalados del calendario romano. Esto puede sugerir la presencia de cierta intencionalidad astronómica del empleo de algunas variationes o triángulos pitagóricos en casos en los que la observación directa de los fenómenos astronómicos no fuera posible (Rodríguez-Antón et alii 2019); pues para obtener una orientación astronómica se ha de tener en cuenta el efecto que produce el horizonte local sobre la visibilidad de los objetos.
Por estos motivos, nuestra propuesta, para nada dogmática, es una opción basada en el uso de la varatio como modus operandi para plasmar algunas orientaciones determinadas sobre estructuras ortogonales. Una técnica que pudo ser utilizada tanto en ámbitos rurales como urbanos, dada la cantidad de bienes que, una vez estudiados, han demostrado orientarse en base a ángulos susceptibles de ser obtenidos mediante opciones de varatio sencillas y una solución geométrica hábil que usa fracciones para representar relaciones entre números enteros.

En el proceso de creación de estos espacios rectangulares o cuadrados, es necesario tener una línea de referencia, siendo nuestra propuesta, como se ha repetido, la que une el polo norte con el sur.

En ocasiones los valores de las ratios de la varatio tienen un margen de diferencia mínima, que no llega a un grado -ver el abanico representado en la Figura $2-$, como $1: 10$, que corresponde a $84,29^{\circ}$ frente a $1: 11$, que son $84,80^{\circ}$ y $1: 12$, de valor $85,24^{\circ}$. Quiere eso decir que en determinados casos las medidas sobre bienes inmuebles pueden tener lecturas "diferentes". Cabe recordar que el margen de actuación, la variabilidad que ofrece la aplicación de la varatio a la que se suma la opción de "ternas pitagóricas", tiene un límite de posibilidades dado que es un modelo estandarizado. En esos casos, habría que valorar cuál es la relación de varatio más plausible (seguramente la más sencilla).

La utilización de ese procedimiento significa que se pueden repetir alineaciones con una misma orientación sumando solares en un mismo territorio, acumulando largas distancias; se pueden copiar orientaciones de lugares lejanos y reproducirlas en nuevos proyectos, simplemente aplicando el mismo juego de fracciones en relación con la línea de referencia, la generadora, que en este caso estimamos que es la meridiana.

La coincidencia de los mínimos principales de las distribuciones de acimuts y los ángulos de varatio que se observan en los diagramas antes expuestos, manifiestan la ausencia de orientaciones que no se corresponden con una varatio; como es el caso de los puntos cardinales y los inter-cardinales; estos últimos se ajus$\tan$ a 1:1. El que las orientaciones no se distribuyan de manera homogénea, sino que aparezcan máximos de acimut bien definidos, además de la coincidencia de algunos de esos máximos con valores de varatio sencillas o de ternas pitagóricas (por ejemplo, pico XII con $1: 3$, Figs. 10 y 11), resaltan la no aleatoriedad de los datos. Además, del resultado del test de KolmogorovSmirnov se extrae que no se puede descartar a priori que ambos juegos de datos -empíricos y varatio- se obtengan de una misma distribución. 
Respecto al empleo de ternas pitagóricas, a raíz de lo extraído de la Figura 12 se podría decir que, si bien no parece que haya muchas ciudades coincidentes con orientaciones cercanas al triángulo 5:12:13 mediante el método anteriormente descrito (5 ciudades), existe un mayor número de ellas cuyos ángulos encajan con la del triángulo pitagórico 3:4:5 (11 ciudades). De hecho, el valor del ángulo del triángulo 5:12:13 coincide con un mínimo de acimut, mientras que los ángulos de la terna 3:4:5, si bien no coinciden con máximos principales, tampoco se corresponden con mínimos. También hay orientaciones que se ajustan a la combinación entre ambos triángulos pitagóricos, como es el caso de Valeria (Fig. 4)

\section{CONCLUSIONES}

La no aleatoriedad mostrada en la distribución de los datos y la aparente coincidencia entre acimuts y proporciones de varatio sencillas en muchos de los lugares estudiados invitan a una inevitable reflexión acerca de la existencia de ciertos criterios geométricos en el diseño y orientación de las ciudades romanas. Si se suma el factor de relativa sencillez en la ejecución y reproducción de ángulos seleccionados en el terreno mediante estas técnicas, se consolida la viabilidad del uso de esta técnica en varios casos. El resultado principal y más llamativo es la coincidencia de los mínimos de acimut con los mínimos de varatio, poniendo de manifiesto una ausencia de orientaciones hacia valores que no coinciden con una relación de varatio o de los dos triángulos pitagóricos más sencillos 3:4:5 y 5:12:13; salvo en el caso de Basti y Gerunda con orientación cardinal.

Además, la predominancia de ángulos equivalentes a varationes/fracciones sencillas, como la 1:3, resulta coherente con las impresiones extraídas de las experiencias prácticas con reproducciones de instrumental romano realizadas por el equipo Sotoer (Orfila et alii 2014d) en las que se vivenciaron, cuantificaron y constataron las dificultades y limitaciones técnicas a las que pudo haberse enfrentado un agrimensor en la Antigüedad. Además, los histogramas muestran máximos de acimut bien definidos, lo que parece apuntar a una predilección por ciertas orientaciones que podría responder a una preferencia por determinadas varationes. A raíz de los resultados obtenidos emergen nuevos interrogantes acerca de la naturaleza de estas orientaciones: ¿qué habría conducido a la elección de una u otra relación de proporcionalidad?, ¿podría explicarse la elección de varationes concretas mediante una correspondencia directa entre estas con direcciones de ortos u ocasos astronómicos, como el del sol en fechas relevantes del calendario romano? Puede decirse que sí, tal y como se ha propuesto en anteriores trabajos (González-García et alii 2014, Rodríguez-Antón et alii 2018b, González-García et alii 2019 y Rodríguez-Antón et alii 2019), así como en comprobaciones con resultados aún inéditos, como pueden ser Italica (Sevilla) o Pollentia (Mallorca), ambas orientadas con relación al solsticio de verano, cada una de ellas con su propio acimut $y$, por tanto, con una orientación en grados que difiere entre ambas, o lo que es lo mismo, con una aplicación de varatio/fracción propia para cada una de las ciudades citadas.

A partir de todo lo expuesto no parece lógico plantear que la plasmación de una orientación se dejara al azar y surge así la necesidad de analizar una muestra más amplia con el fin de comprender y esclarecer qué hay detrás de estos resultados. Esto es, observar si se repiten patrones correspondientes a ciertas varationes en otras regiones, si predominan unas frente a otras, y las motivaciones subyacentes en cada una de esas alineaciones, ya sean de tipo práctico o cosmológico.

\section{BIBLIOGRAFÍA}

Adam, J. P. 1982: "Groma et chorobate. Exercices de topographie antique", Mélanges de l'École Française de Rome, Antiquité XCIV, 1003-1029.

Assénat, M. 2006: Cadastre et romanisation dans la cité antique de Nîmes, Revue Archéologique de Narbonnaise, suppl. XXXVI, Montpellier.

Baratta, G. 2006: "Misurari per mestiere", M. G. Angeli Bertinelli y A. Donati (eds.), Misurare il tempo. Misurare lo spazio, Epigrafia e Antichità XXV, Atti del Colloquio AIEGL-Borghesi 2005, Faenza, 233-260.

Baxter, M. J. 2015: Exploratory Multivariate Analysis in Archaeology, EWP Foundations of Archaeology, Clinton Corners, New York.

Behrends, O. 1992: "Bodenhoheit und privates Bodeneigentum im Grenzwesen Roms", Die Römische Feldmesskunst, 192-280.

Belmonte, J. A. y González-García, A. C. 2013: "Metodología y fundamento de las observaciones arqueoastronómicas”, F. Criado-Boado, A. Martínez Cortizas y M. V. García Quintela, Petroglifos, paleoambiente y paisaje. Estudios interdisciplinares del arte rupestre de Campo Lameiro, Madrid, 59-64.

Belmonte, J. A., González-García, A. C., Polcaro, A., Rodríguez-Antón, A. y Shaltout, M. 2016: “Orientatio ad Sidera (OAS): Highlights of a decade of archaeoastronomical research in the Mediterra- 
nean region and beyond", Mediterranean Archaeology and Archaeometry XVI.4, 93-101.

Belmonte, J. A. y Hoskin, M. 2002: “África del Norte antes del Islam", Reflejo del cosmos: atlas del Mediterráneo Antiguo, Madrid, 297-283.

Belmonte, J. A., Tejera Gaspar, A., Perera Betancort, A. y Marrero, R. 2006: "On the orientation of preislamic temples in North Africa: a re-appraisal (new data in Africa Proconsularis)", Mediterranean Archaeology and Archaeometry VI.3, 77-85.

Berrocal-Rangel, L., de la Barrera, J. L. y Caso, R. 2014: "El santuario de Nertobriga Concordia Iulia: una aportación al conocimiento de los rituales de fundación", Journal of Roman Archaeology XXVII, 83-108.

Bouma, J. 1993: Marcus Iunius Nypsus. Fluminis Varatio, Limitis Repositio. Introduction, Text, Translation and Commentary, Studien zur klassischen Philologie LXXVII, Frankfurt/Main.

Campbell, J. B. 1996: "Shaping the rural environment: surveyors in Ancient Rome", The Journal of Roman Studies LXXXVI, 74-99.

Campbell, J. B. 2000: The writings of the Roman Land surveyors. Introduction, Text, Translation and Commentary, Journal of Roman Studies Monograph XIX, London.

Capone, G. 1991: “L'Orientazione solstiziale dell'antica città di Alatri', Colloquio Int. Archeologia e Astronomia, Roma, 60-65.

Castillo Pascual, M. ${ }^{a}$ J. 1993: "Agrimensura y agrimensores: el Corpus agrimensorum romanorum", Hispania Antiqua XVII, 143-158.

Castillo Pascual, M. ${ }^{\text {a }}$ J. 1996: Espacio de orden: El modelo gromático-romano de ordenación del territorio, Logroño.

Chevallier, R. 1967: "Sur les traces des arpenteurs romains", Caesarodunum II, Orleans-Tours.

Chouquer, G. 2004: "Une nouvelle interprétation du Corpus des Gromatici Veteres", Agri Centuriati 1, 44-56.

Chouquer, G. y Favory, F. 1992: Les Arpenteurs romain. Théorie et Practique, Paris.

Chouquer, G. y Favory, F. 2001: L'arpentage romain. Histoire des textes-Droit-Techniques, Paris.

Clavel-Lévêque, M. 1992: "Centuriation, géométrie et harmonie. Le cas du Biterrois", J. Y. Guillaumin (dir.), Mathématiques dans l'Antiquité, SaintÉtienne, 162-184.

Costa, V. y Orfila, M. 2014: "Creación física de gnómones”, M. Orfila, E. Chávez-Álvarez y E. H. Sánchez (eds.), La orientación de las estructuras ortogonales de nueva planta en época romana. De la varatio y sus variaciones, Granada, 129-155.

Dall'Aglio, P. L. 2004: "Perché studiare la centuriazione”, Agri Centuriati I, 17-21.
Decramer, L. R. y Hilton, R. 1996: "Le problème de la grande centuriation de l'Africa Nova. Esquisse d'une solution", Cahiers de la Tunisie XLIX, CLXXIV, 43-95.

Decramer, L. R., Hilton, R., Lapierre, L. y Plas, A. 2003: "Le cadastre romain d'Orange", Archéologie CDIV, 36-46.

Decramer, L. R., Hilton, R. Lapierre, L. y Plas, A. 2006: "La grande carte de la colonie romaine d'Orange", A. Gonzales y J.-Y. Guillaumin, (eds.), Autour des Libri coloniarum. Colonisation et colonies dans le monde romain, Franche-Comté, 93-114.

Dilke, O. A. W. 1971: The Roman Land Surveyors: an introduction to the Agrimensores, New York.

Eckstein, A. 1979: "The Foundation Day of Roman Coloniae", California Studies in Classical Antiquity XII, 85-97.

Equipo Sotoer 2014: "La técnica de la varatio en el mundo antiguo", M. Orfila, E. Chávez-Álvarez y E. H. Sánchez (eds.), La orientación de las estructuras ortogonales de nueva planta en época romana. De la varatio y sus variaciones, Granada, 72-85.

Espinosa Espinosa, D. y González-García, A. C. 2017: "a.d. VIIII Kalendas Octobres, dies natalis Augusti. Some considerations on the Astronomical Orientation of Roman Cologne and the Imperial Cult", Numen LXIV, 545-567.

Esteban, C., Belmonte, J. A., Perera Betancourt, M. A., Marrero, R., y Jiménez González, J. J. 2001: “Orientations of Pre-Islamic Temples of Northwest Africa", Journal for the History of Astronomy, Archaeoastronomy Supplement XXXII, 65-84.

Ferrar, M. J. y Richardson, A. 2003: The Roman Survey of Britain, Oxford.

Ferro, L. y Magli, G. 2012: "The astronomical orientation of the urban plan of Alexandria", Oxford Journal of Archaeology XXXI, 381-389.

González-García, A. C. y Magli, G. 2015: "Roman City Planning and Spatial Organization", C. L. N. Ruggles (ed.), Handbook of Archaeoastronomy and Etnoastronomy, New York, 1643-1650.

González-García A. C., Noguera Celdrán J. M., Belmonte Avilés J. A., Rodríguez-Antón, A., Ruiz Valderas, E., Madrid Balanza, M. J., Zamora E. y Bonnet Casciaro, J. 2015: "Orientatio ad sidera: Astronomía y paisaje urbano en Qart Hadašt/Carthago Nova", Zephyrus LV, 141-162.

González-García, A. C., Rodríguez-Antón, A. y Belmonte, J. A. 2014: "The Orientation of Roman Towns in Hispania: Preliminary Results", Mediterranean Archaeology and Archaeometry XIV.3, 107-119. 
González-García, A. C., Rodríguez-Antón, A., Espinosa-Espinosa, D., García-Quintela, M. V. y Belmonte, J. A. 2019: "Establishing a New Order: The orientation of Roman Towns Built in the Age of Agustus”, G. Magli, A. C. González-García, J. Belmonte Avilés y E. Antonello (eds.), Archaeoastronomy in the Roman World, 89-102.

Gotarelli, A. 2003: "Modello cosmologico, rito di fondazione e sistemi di orientazione rituale. La connessione solare", Ocnus XI, 151-170.

Gotarelli, A. 2004: "Templum Solare e città fondata. La connessione astronomica della forma urbana della città etrusca di Marzabotto (III)", A. Sassatelli y E. Govi (eds.), Culti, Forma Urbana e artigianato a Marzabotto. Nuove prospettive di ricerca, Bologna, 101-138.

Gros, P. 1976: "Nombres irrationnels et nombres parfaits chez Vitruve", Mémoires de l'Ecole Française de Roma, Antiquité LXXXVIII.2, 669-704.

Guy, M. 1993: "Les orientations des parcellaires quadrillés", Revue archéologique de Narbonnaise XXVI, 57-68.

Hannah, R. y Magli, G. 2011: "The role of the sun in the Pantheon's design and meaning", Numen LVIII.4, 486-513.

Haselberger, L. 1999: "Geometrie der winde, windige geometrie: Städtebau nach Vitruv und Aristophanes“, E. L. Schwandner y K. Rheidt (eds.), Stad und Umland. Neue Ergebnisse der archäologischen Bau- und Siedlungsforschung. Diskussionen zur Archäologischen Bauforschung Band VII, Mainz am Rhein, 90-100.

Lanciano, N. y Virgili, P. 2016: "The urban set of the Pantheon and the Mausoleum of Augustus in Rome, between architectural and astronomical symbolism", Mediterranean Archaeology and Archaeometry XVI.4, 249-255.

Le Gall, J. 1975: "Les Romains et l'orientation solaire", Mélanges de l'École Française de Rome, Antiquité LXXXVII, 287-320.

Lewis, M. J. T. 2001: Surveying Instruments in Ancient Greece and Rome, Cambridge.

Magli, G. 2008: "On the Orientation of Roman Towns in Italy", Oxford Journal of Archaeology XXI.6, 63-71.

Neugebauer, O. 1969: The exact sciences in Antiquity, New York.

Orfila, M. 2009: "Diseño del trazado urbano del foro de Pollentia”, J. M. Noguera (ed.), Fora Hispaniae. Paisaje urbano, arquitectura, programas decorativos y culto imperial en los foros de las ciudades hispanorromanas, Monografías 3, Murcia, 203-16.

Orfila, M. 2011: "Arqueología Experimental aplicada al urbanismo. Un procedimiento para trazar y orientar estructuras ortogonales en época romana”, A. Morgado, J. Baena y D. García (eds.), $L a$ investigación experimental aplicada a la Arqueología, Granada, 289-297.

Orfila, M. 2012: "Un posible sistema para orientar estructuras de trazado ortogonal en época clásica. El caso de Pollentia (Mallorca, España)". Geographia Antiqua XX-XXI, 123-136.

Orfila, M. 2014a: "La creación de infraestructuras públicas en época romana”, M. Orfila, E. ChávezÁlvarez y E. H. Sánchez (eds.), La orientación de las estructuras ortogonales de nueva planta en época romana. De la varatio y sus variaciones, Granada, 57-67.

Orfila, M. 2014b: "Los triángulos en el mundo antiguo”, M. Orfila, E. Chávez-Álvarez y E. H. Sánchez (eds.), La orientación de las estructuras ortogonales de nueva planta en época romana. De la varatio y sus variaciones, Granada, 67-69.

Orfila, M. y Chávez-Álvarez, E. 2014: "La línea norte -meridiano-. Como generadora de los trazados", M. Orfila, E. Chávez-Álvarez y E. H. Sánchez (eds.), La orientación de las estructuras ortogonales de nueva planta en época romana. De la varatio y sus variaciones, Granada, 112-122.

Orfila, M., Chávez-Álvarez, E. e Iglesia, M. A. de la 2014a: "La localización del norte a través de un gnomon: procedimiento", M. Orfila, E. ChávezÁlvarez y E. H. Sánchez (eds.), La orientación de las estructuras ortogonales de nueva planta en época romana. De la varatio y sus variaciones, Granada, 123-126.

Orfila, M., Chávez-Álvarez, E. e Iglesia, M. A. de la 2014b: "El instrumental y el aparataje utilizado en época antigua en tareas de planificación y construcción”, M. Orfila, E. Chávez-Álvarez y E. H. Sánchez (eds.), La orientación de las estructuras ortogonales de nueva planta en época romana. De la varatio y sus variaciones, Granada, 126-129.

Orfila, M., Chávez-Álvarez, E., Iglesia M. A. de la y Sánchez, E. H. 2014c: "El gnomon y los triángulos pitagóricos como instrumentos de planificación básicos en el urbanismo romano: su aplicación sobre yacimientos arqueológicos hispanos", J. M. Álvarez Martínez, T. Nogales Basarrate e I. Rodà de Llanza (eds.), XVIII CIAC: Centro y periferia en el mundo clásico, V. I, Mérida, 773-776.

Orfila, M., Moranta, L., Chávez-Álvarez, E., Iglesia M. A. de la y Sánchez, E. H. 2014d: "Un caso particular de la varatio. El uso de ternas pitagóricas", M. Orfila, E. Chávez-Álvarez y E. H. Sánchez (eds.), La orientación de las estructuras ortogonales de nueva planta en época romana. De la varatio y sus variaciones, Granada, 86-109. 
Orfila Pons, M., Chávez-Álvarez, M. E. y Sánchez, E. H. 2017a: "Fundaciones en época romana. De lo intangible a lo tangible. ¿Cuándo, por qué, dónde, cómo, simbología?", Sagvntvm-PLAV XIX, 265-276.

Orfila Pons, M., Chávez-Álvarez, M. E. y Sánchez, E. H. 2017b: "Urbanizar en época romana: ritualidad y practicidad. Propuesta de un procedimiento homologado de ejecución", Spal XXVI, 113-134.

Orfila, M. y Moranta, L. 2001: "Estudio del trazado regulador del Foro de Pollentia (Alcúdia, Mallorca)", Archivo Español de Arqueología LXXIV, 209-232.

Pérez, A. 1995: "Les cadastres antiques en Narbonnaise occidentale. Essai sur la politique coloniale romaine en Gaule du sud ( $\mathrm{II}^{\mathrm{e}}$ s. av. J.-C.-II ${ }^{\mathrm{e}}$ s. ap. J.-C.)", Revue Archéologique de Narbonnaise, suppl. XXIX, Paris.

Peterson, J. W. M. 1992: "Trigonometry in Roman cadastre", J.-Y. Guillaumin (ed.), Mathématiques dans l'Antiquité, St.-Étiene, 185-203.

Peterson, J. W. M. 2007: "Random Orientation of Roman Camps", Oxford Journal of Archaeology XXVI.1, 103-108.

Richardson, A. 2005: "The orientation of Roman Camps and Fores", Oxford Journal of Archaeology XXIV.4, 415-426.

Rodríguez-Antón, A., Belmonte, J. A. y GonzálezGarcía, A. C. 2016a: "Romans in the Near East: the orientation of Roman Settlements in presentday Jordan", Mediterranean Archaeology and Archaeometry XVI.4, 153-160.

Rodríguez-Antón, A., González-García, A. C. y Belmonte, J. A. 2016b: "Orientation of Roman Camps and Forts in Britannia: Reconsidering Alan Richardson's Works", F. Silva, K. Malville, T. Lomsdalen y F. Ventura (eds.), The Materiality of the Sky, Wales, 135-143.

Rodríguez-Antón, A., González-García, A. C. y Belmonte, J. A. 2018a: "Astronomy in Roman urbanism: a statistical analysis of the orientation of Roman towns in the Iberian Peninsula", Journal for the History of Astronomy 49.3, 363-387.

Rodríguez-Antón, A., González-García, A. C. y Belmonte, J. A. 2018b: "An archaeoastronomical approach to Roman urbanism. Orientation of Roman settlements across the Empire", Mediterranean Archaeology and Archaeometry XVIII.4, 89-95.

Rodríguez-Antón, A., Orfila, M., González-García, A. C. y Belmonte, J. A. 2019: "The Uaratio and its Possible Use in the Roman Urban Planning to $\mathrm{Ob}$ tain Astronomical Orientations", G. Magli, A. C. González-García, J. Belmonte Aviles y E. Antonello (eds.), Archaeoastronomy in the Roman World, 103-118.
Romano, G. 1991: "Orientamenti magnetici e astronomici nelle mappe archeologiche”, M. Santi (ed.), Colloquio Internazionale Archeologia e Astronomia, Rivista dei Archeologia Supplementi 9, Roma, 23-29.

Rosada, G. 1991: "Divisione agraria, orientamiento e suolo", M. Santi, Archeologia e astronomia, Colloquio Internazionale, Rivista dei Archeologia IX, 88-95.

Roskams, S. 2001: Excavation, Cambridge Manuals in Archaeology, Cambridge.

Rossella Filippi, M. 1983: "Le procedure: le operazioni preliminari”, R. Bussi y V. Vandelli (eds.), Misurare la terra: centuriazione e coloni nel mondo romano, Modena, 124-127.

Roth-Congès, A. 1996: "Modalités pratiques d'implantation des cadastres romains: quelques aspects (Quintarios claudere. Perpendere. Cultellare. Varare: la construction des cadastres sur une diagonale et ses traces dans le Corpus Agrimensorum)", Mélanges de l'Ecole Française de Rome, Antiquité CVIII.1, 299-422.

Roth-Congès, A. 1998: "La 'varation dans les territoires' de M. Iunius Nypsius: une application des propriétés des triangles semblables à la construction cadastrale", M. Clavel-Lévêque y A. Vignot (eds.) Cité et territoire II, París, 165-184.

Roth-Congès, A. 2001: "Instruments et techniques d'arpentage", G. Chouquer y F. Favory (eds.), L'arpentage romain, Paris, 64-70; 79-94.

Ruggles, C. L. N. 2015a: "Long-Term Changes in the Appearance of the Sky”, C. L. N. Ruggles (ed.), Handbook of Archaeoastronomy and Etnoastronomy, New York, 473-482.

Ruggles, C. L. N. 2015b: "Basic concepts of positional astronomy", C. L. N. Ruggles (ed.), Handbook of Archaeoastronomy and Etnoastronomy, New York, 459-72.

Salvatori, M. 2006: Manuale di Metrologia per architetti studiosi di storia dell'architettura ed archeologi, Napoli.

Sánchez, E. y Marín, P. 2014: "Los chequeos empíricos sobre el terreno”, M. Orfila, E. Chávez-Álvarez y E. H. Sánchez (eds.), La orientación de las estructuras ortogonales de nueva planta en época romana. De la varatio y sus variaciones, Granada, 157-187.

Toro, A. 1983: “Strumenti relativi all'agrimensura”, R. Bussi y V. Vandelli (eds.), Misurare la terra: centuriazione e coloni nel mondo romano, Modena, 31-37.

Toro, A., 1985: "Strumenti relativi all'agrimensura", R. Bussi y V. Vandelli (eds.), Misurare la terra: centuriazione e coloni nel mondo romano. Città, 
146 ORFILA PONS, RODRÍGUEZ-ANTÓN, CHÁVEZ-ÁLVAREZ, GONZÁLEZ-GARCÍA, SÁNCHEZ LÓPEZ, BELMONTE

agricoltura, commercio: materiali da Roma e dal suburbi, Roma, 31-37.

Thulin, C. 1913: Corpvs Agrimensorvm Romanorvm. Opvscvla Agrimensorvm Vetervm, Studgard.

Ventura, A. 2008: "La ortogonalidad como componente ritual: diseño urbanístico de una colonia romana", P. León (coord.), Arte Romano de la Bética. Arquitectura y urbanismo, Sevilla, 64-69.
Welfare, H. y Swan, V. 1995: Roman camps in England: The Field Archaeology, London.

Recibido: 30-04-2019

Aceptado: 16-09-2019 